US Army Corps

of Engineers ${ }_{\circledast}$

Engineer Research and

Development Center

\title{
Analysis of Snow Water Equivalent Annual Maxima in the Upper Connecticut River Basin Using a Max-Stable Spatial Process Model
}

Brian Skahill, Joseph Kanney, and Meredith Carr

April 2020 
The US Army Engineer Research and Development Center (ERDC) solves the nation's toughest engineering and environmental challenges. ERDC develops innovative solutions in civil and military engineering, geospatial sciences, water resources, and environmental sciences for the Army, the Department of Defense, civilian agencies, and our nation's public good. Find out more at www.erdc.usace.army.mil.

To search for other technical reports published by ERDC, visit the ERDC online library at http://acwc.sdp.sirsi.net/client/default. 


\title{
Analysis of Snow Water Equivalent Annual Maxima in the Upper Connecticut River Basin Using a Max-Stable Spatial Process Model
}

\author{
Brian Skahill \\ Coastal and Hydraulics Laboratory \\ US Army Engineer Research and Development Center \\ 3909 Halls Ferry Road \\ Vicksburg, MS 39180-6199 \\ Joseph Kanney and Meredith Carr \\ US Nuclear Regulatory Commission \\ Office of Nuclear Regulatory Research \\ 11555 Rockville Pike \\ Rockville, MD 20852-2738, Mail Stop TWFN-10A12
}

Final report

Approved for public release; distribution is unlimited

Prepared for US Nuclear Regulatory Commission

11555 Rockville Pike

Rockville, MD 20852-2738

Under US NRC; MIPR Agreement No. NRC-HQ-60-14-I-0022 


\section{Abstract}

Recent advances from the science of spatial extremes and model regularization were applied to develop areal-based extremes of snow water equivalent (SWE) data for the upper Connecticut River Basin.

Development of areal-based SWE exceedance probability estimates are of relevance for cool season probabilistic flood hazard analyses (PFHA). The approach profiled in this case study is applicable for other hydrometeorological variables of relevance to PFHA. The methodology conforms with Extreme Value Theory (EVT) for the analysis of spatial extremes; hence, there is a firm theoretical basis for extrapolation. Trend surface development is guided by EVT theory and recent advances for regularizing general linear models. $\mathrm{R}$, a free software environment for statistical computing and graphics, and QGIS, a free and open-source geographic information system, were the primary tools used for product development and delivery. The following R software packages were primarily used during project execution: evd, Glmnet, maps, raster, rgdal, SDMTools, sp, and SpatialExtremes. R software packages exist in the public domain and support PFHA analyses of varying complexities. Their application herein is not an endorsement or recommendation. It is recommended that one would need to evaluate any particular R software package regarding its suitability for use for any specific application.

DISCLAIMER: The contents of this report are not to be used for advertising, publication, or promotional purposes. Citation of trade names does not constitute an official endorsement or approval of the use of such commercial products. All product names and trademarks cited are the property of their respective owners. The findings of this report are not to be construed as an official Department of the Army position unless so designated by other authorized documents. 


\section{Contents}

Abstract................................................................................................................................... if

Figures and Tables......................................................................................................................

Preface

1 Introduction .......................................................................................................... 1

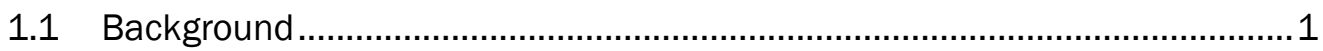

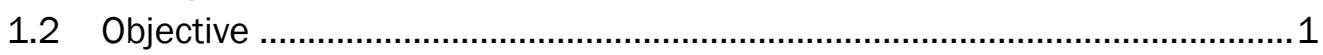

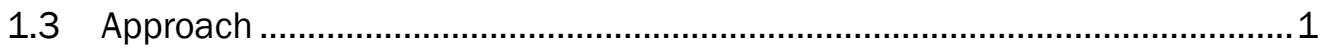

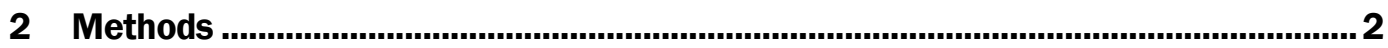

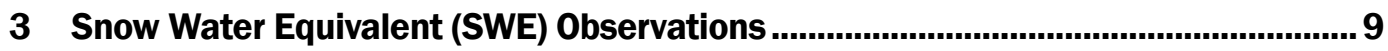

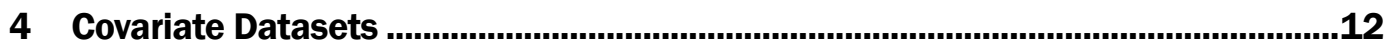

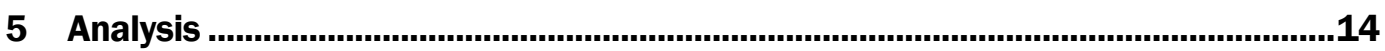

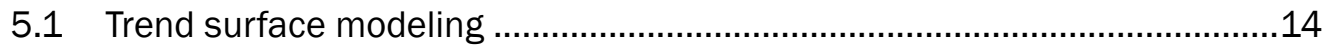

5.2 Simple max-stable model selection........................................................ 23

5.3 General max-stable model development and application ............................24

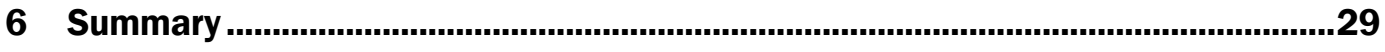

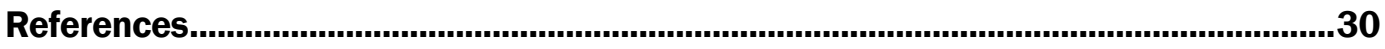

Acronyms and Abbreviations.................................................................................33

Report Documentation Page 


\section{Figures and Tables}

\section{Figures}

Figure 1. Five independent realizations of the Smith, Schlather, Brown-Resnick, and Extremal-t processes on $\mathrm{X}=0,10$ (Ribatet 2015)

Figure 2. One realization of the Smith process with $\Sigma$ given by the identity matrix, Schlather process with $\rho \mathrm{h}=\mathrm{exp}-\mathrm{h} 21.5$, and the extremal-t process, with $v=5$ and $\rho \mathrm{h}=\mathrm{exp}-\mathrm{h} 21.5$, on a $250 \times 250$ grid (Ribatet et al. 2015).

Figure 3. SWE observation site locations overlain on a digital elevation model (DEM) (SWE networks: $=$ =HBEF; $=$ =SCAN; $=$ =NHDES; $=$ =USACE; DEM data source: PRISM 30 year normal (Norm81m dataset) (http://www.prism.oregonstate.edu/normals/).

Figure 4. Mean of the SWE annual maxima (inches) for each SWE observation location overlain on a digital elevation model (DEM) (DEM data source: PRISM 30 year normal (Norm81m dataset) (http://www.prism.oregonstate.edu/normals/).

Figure 5. Spatial distribution of the at-site extremal model parameters (a) $\mu$, (b) $\sigma$, and (c) $\xi$, respectively.

Figure 6. Summary plots associated with three independent elastic net applications to build trend surfaces for (a) $\mu(\mathrm{x})$, (b) $\sigma(\mathrm{x})$, and (c) $\xi \mathrm{x}$, respectively.

Figure 7. Summary plots associated with three independent elastic net applications to build trend surfaces for (a) $\mu(\mathrm{x})$, (b) $\sigma(\mathrm{x})$, and (c) $\xi \mathrm{x}$, respectively, that underscore the tradeoff between model fit and complexity.

Figure 8. Plot of information criterion scores for the defined, fitted, and evaluated spatial GEV models (model naming convention: "Mod" + " $\xi(x)$ Model Index" + “ $\sigma(\mathrm{x})$ Model Index" + “ $\mu \mathrm{x}$ Model Index").

Figure 9. Comparisons of the fitted spatial GEV trend surface models with corresponding at-site estimates ( $\mu=$ GEV location; $\sigma=$ GEV scale; $\xi=$ GEV shape; Model = spatial model; MLE = at-site maximum likelihood estimate).

Figure 10. Summary measure of fit for the selected Extremal-t simple max-stable process ( $\theta$ is the extremal coefficient function; $h$ is normalized distance).

Figure 11. Summary diagnostic of fitted general max-stable process model.

Figure 12. Comparisons of the fitted general max-stable model with corresponding at-site estimates ( $\mu=$ GEV location; $\sigma=$ GEV scale; $\xi=$ GEV shape; Model = general max-stable spatial model; $M L E=$ at-site maximum likelihood estimate).

Figure 13. Fitted trend surfaces for (a) $\mu(\mathrm{x})$, (b) $\sigma(\mathrm{x})$, and (c) $\xi \mathrm{x}$, and (d) the 100year pointwise return levels of SWE AM, in inches, for the upper CRB.

Figure 14. Two independent copies of the fitted max-stable model of SWE AM, in inches, for the White River Basin in the upper CRB

Figure 15. Computed histogram of mean basin SWE AM, in inches, for the White River Basin of the upper Connecticut River Basin. 


\section{Tables}

Table 1. Number of observation sites and annual maxima associated with each SWE network.

Table 2. Summary listing of the 135 collected and processed base covariates. Monthly, annual, and seasonal (average of November - April monthlies) products were collected and processed for the climatology datasets (PRECIP, TMEAN, TMAX, TMIN, TDMEAN, DLWRF, DSWRF, EWIND, NWIND).

Table 3. Summary of elastic net derived trend surfaces for $\mu(\mathrm{x})$ extracted for spatial GEV model definition, fitting, and evaluation ( $*=$ best regularizing $\lambda$ model; $* *=$ minimizing $\lambda$ model).

Table 4. Summary of elastic net derived trend surfaces for $\sigma(\mathrm{x})$ extracted for spatial GEV model definition, fitting, and evaluation ( $*=$ best regularizing $\lambda$ model; $* *=$ minimizing $\lambda$ model).

Table 5. Summary of elastic net derived trend surfaces for $\xi(x)$ extracted for spatial GEV model definition, fitting, and evaluation. The minimizing model with a mean MSE value of 0.0571 was at $\lambda=0.0272243$ with 6 non-zero covariates, and the best regularizing model, with a mean MSE equal to 0.07 , was at $\lambda=$ 0.1049096 , with two non-zero covariates.

Table 6. Areal-based exceedance probability estimates of the SNODAS modeled mean SWE AM for the White River. 


\section{Preface}

This study was conducted for the US Nuclear Regulatory Commission under US NRC, MIPR Agreement No. NRC-HQ-60-14-I-0022. The technical monitor was Dr. Joseph Kanney.

The work was performed by the Hydrologic Systems Branch of the Flood and Storm Protection Division, US Army Engineer Research and Development Center, Coastal and Hydraulics Laboratory (ERDC-CHL). At the time of publication, Dr. Hwai-Ping Cheng was Branch Chief; Dr. Cary Talbot was Division Chief; and Dr. Julie Rosati was the Technical Director for Flood Risk Management. The Deputy Director of ERDC-CHL was Mr. Jeffrey R. Eckstein, and Dr. Ty V. Wamsley was the Director.

The Commander of ERDC was COL Teresa A. Schlosser, and the Director was Dr. David W. Pittman. 


\section{Introduction}

\subsection{Background}

Simulation of cool season hydrologic processes such as snowmelt-induced flooding often involves using observed snow water equivalent (SWE). SWE is the amount of water contained within the snowpack. While it is often measured at discrete points in space to support water management operations, it can also be processed much like precipitation or flow data to support risk-informed hydrologic planning. SWE values are often specified on an areal basis (e.g., elevation bands or by sub-basin when they are used to support hydrologic simulation of cool season processes). Hence, related risk-informed hydrologic analyses require areal-based exceedance probabilities for SWE.

\subsection{Objective}

This case study demonstrates the use of max-stable spatial processes to calculate pointwise and areal-based exceedance probabilities for SWE.

\subsection{Approach}

Recent advances have demonstrated the capacity to efficiently, flexibly, and credibly model spatial extremes of pointwise maxima (e.g., Davison and Gholamrezaee 2011; Reich and Shaby 2012; Olinda et al. 2014;

Stephenson et al. 2016; Nicolet et al. 2015; Blanchet and Davison 2011). Each of these studies involved application of max-stable processes, the stochastic process extension of extreme value theory (EVT). With their application, one can not only compute pointwise return level maps, but also by modeling the joint distribution, of SWE for the case study application presented herein, more complex areal-based exceedance probabilities such as

$$
\operatorname{Pr}\left\{\int_{\mathcal{B}} \Upsilon(x) d x>z_{\text {crit }}\right\}
$$

where $\Upsilon(x), \mathcal{B}$, and $z_{\text {crit }}$ denote the joint distribution, an arbitrary area within the computational domain (e.g., a sub-basin, and a critical quantity greater than zero, respectively [Ribatet et al. 2015]). 


\section{Methods}

This section is a succinct practice-oriented summary of max-stable processes for the calculation of spatial extremes. Ribatet (2017, 2013), Ribatet et al. (2015), Davison et al. (2012), and Cooley et al. (2012) provide comprehensive technical descriptions of max-stable processes. From univariate EVT, it is known that if normalizing sequences exist for a sequence of independent and identically distributed random variables such that the rescaled variables have a nondegenerate limiting distribution, then the distribution is max-stable and, moreover, that a distribution is max-stable if and only if it is the generalized extreme value (GEV) distribution (Coles 2001). Mathematical nondegenerate limit law expressions of max-stability, comparable in form to the univariate case, that involve the existence of normalizing sequences also exist in the multivariate and stochastic process settings (Ribatet 2017). Univariate EVT results guarantee that the marginal distribution of max-stable processes are GEV distributed with possibly different parameters by location (Ribatet et al. 2015; Ribatet 2017).

As previously mentioned, this study applies a max-stable spatial process wherein just as with univariate EVT this limiting process is used to model the partial maxima. It is convenient and without any loss of generality to define a simple max-stable process, denoted by $\left\{Z(x): x \in X \subset \mathbb{R}^{d}, d \geq 1\right\}$, to be with fixed unit Fréchet, rather than spatially variable GEV, margins. In this case, $\operatorname{Pr}\{Z(x) \leq z\}=\exp (-1 / z) \forall x \in \mathcal{X}$ and $z>0$. de Haan (1984) introduced the spectral representation of a simple max-stable process. It states that $\{Z(x): x \in \mathcal{X}\} \equiv\left\{\max _{i \geq 1} \zeta_{i} Y_{i}(x): x \in \mathcal{X}\right\}$, where $\left\{\zeta_{i}: i \geq 1\right\}$ is a Poisson point process on $(0, \infty)$ with intensity measure $\zeta^{-2} d \zeta$ and $\left\{Y_{i}(x): x \in \mathcal{X}, i \geq 1\right\}$ a sequence of independent copies of a non-negative stochastic process $\{Y(x): x \in \mathcal{X}\} \ni \mathbb{E}\{Y(x)\}=1 \forall x \in \mathcal{X}$ (三 indicates equality in distribution). The spectral representation of a max-stable process introduced by de Haan (1984) has resulted in the subsequent development of usable parametric models for spatial extremes, with different distributional assumptions for $\{Y(x): x \in \mathcal{X}\}$ resulting in different max-stable models.

The famous Smith process (Smith 1990), also known as the Gaussian

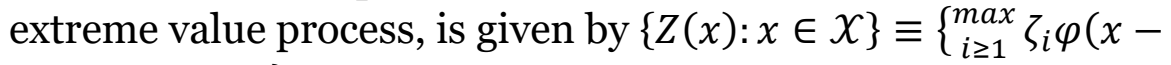
$\left.\left.U_{i} ; 0, \Sigma\right): x \in \mathcal{X}\right\}$, where $\varphi(\cdot ; 0, \Sigma)$ is the $d$-variable Gaussian density with 
zero mean and covariance $\Sigma$ and $\left\{\left(\zeta_{i}, U_{i}\right): i \geq 1\right\}$ are the points of a Poisson process on $(0, \infty) \times \mathbb{R}^{d}$ with intensity measure $\zeta^{-2} d \zeta d u$ (Ribatet et al. 2015; Ribatet 2017). The Schlather process (Schlather 2002), also known as the extremal Gaussian process, is given by $\{Z(x): x \in X\} \equiv$ $\left\{\sqrt{2 \pi} \max _{i \geq 1} \zeta_{i} \max \left(0, \varepsilon_{i}(x)\right): x \in X\right\}$, where $\left\{\varepsilon_{i}(x): x \in X\right\}$ are independent copies of a stationary Gaussian process with correlation function $\rho$ (Ribatet et al. 2015; Ribatet 2017). The R software package SpatialExtremes implements the Bessel, Cauchy, generalized Cauchy, powered exponential, and Whittle-Matern correlation functions (Ribatet 2018). The Brown-Resnick process (Kabluchko et al. 2009; Brown and

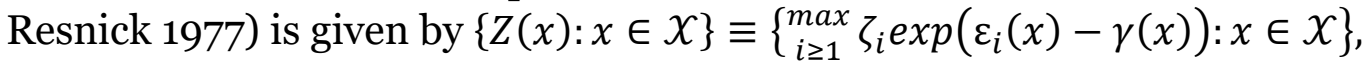
where $\left\{\varepsilon_{i}(x): x \in \mathcal{X}\right\}$ are independent copies of a zero mean Gaussian process with stationary increments and semi-variogram $\gamma(x)=$ $\operatorname{Var}\{\varepsilon(x+h)-\varepsilon(x)\} / 2$ (Ribatet et al. 2015; Ribatet 2017). The Smith model is in fact a special case of the Brown-Resnick process. The extremal$\mathrm{t}$ process (Opitz 2013) is given by $\{Z(x): x \in X\} \equiv$ $\left\{c_{\nu} \max _{i \geq 1} \zeta_{i} \max \left(0, \varepsilon_{i}(x)\right)^{v}: x \in X\right\}$, where $\left\{\varepsilon_{i}(x): x \in X\right\}$ are independent copies of a stationary Gaussian process with correlation function $\rho$ and $c_{v}=\sqrt{\pi} 2^{-(v-2) / 2} \Gamma\left(\frac{v+1}{2}\right)^{-1}$ where $\Gamma$ is the gamma function (Ribatet et al. 2015; Ribatet 2017). The Schlather process is a special case of the extremal-t process with $v=1$ (Ribatet et al. 2015; Ribatet 2017).

Figure 1 includes separate plots of the Smith process with variance equal to 2, Schlather process with $\rho(h)=\exp \left\{-(h / 3)^{1}\right\}$, Brown-Resnick process with $\gamma(x)=(h / 3)^{1}$, and extremal-t process with $v=4$ and $\rho(h)=$ $\exp \left\{-(h / 3)^{1}\right\}$, with each plot depicting five independent realizations of each respective process on $X=[0,10]$ (Ribatet 2015). Figure 2 depicts one realization of the Smith process with $\Sigma$ given by the identity matrix, Schlather process with $\rho(h)=\exp \left\{-(h / 2)^{1.5}\right\}$, and the extremal-t process, with $v=5$ and $\rho(h)=\exp \left\{-(h / 2)^{1.5}\right\}$, for a $250 \times 250$ grid of $\mathcal{X}=[0,10] \times$ $[0,10]$ (Ribatet et al. 2015). The Smith process realizations depicted in Figures 1 and 2 underscore the reports that it produces artificial surfaces that are too smooth for many practical applications (Ribatet et al. 2015; Ribatet 2017). The Schlather process realizations depicted in Figures 1 and 2 underscore summaries emphasizing that it tends to produce, unrealistically, due to a limitation, larger areas with the largest values in comparison with the extremal-t and Brown-Resnick processes (Ribatet et al. 2015). The Brown-Resnick process is known to be difficult to work with (Ribatet et al. 2015). Nicolet et al. (2015) reported better performance for the extremal-t 
process relative to other available max-stable processes in their study of the dependence structure of extreme snowfall in the French Alps.

Figure 1. Five independent realizations of the Smith, Schlather, Brown-Resnick, and Extremal-t processes on $X=[0,10]$ (Ribatet 2015).

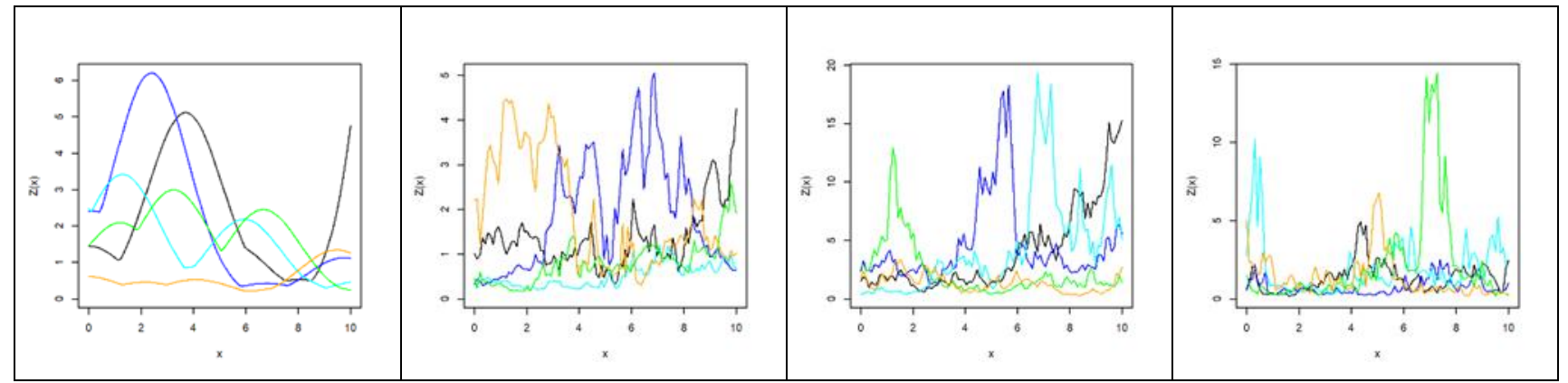

Figure 2. One realization of the Smith process with $\Sigma$ given by the identity matrix, Schlather process with $\rho(h)=\exp \left\{-(\boldsymbol{h} / 2)^{1.5}\right\}$, and the extremal-t process, with $\boldsymbol{v}=\mathbf{5}$ and $\boldsymbol{\rho}(\boldsymbol{h})=$ $\exp \left\{-(h / 2)^{1.5}\right\}$, on a $250 \times 250$ grid (Ribatet et al. 2015).

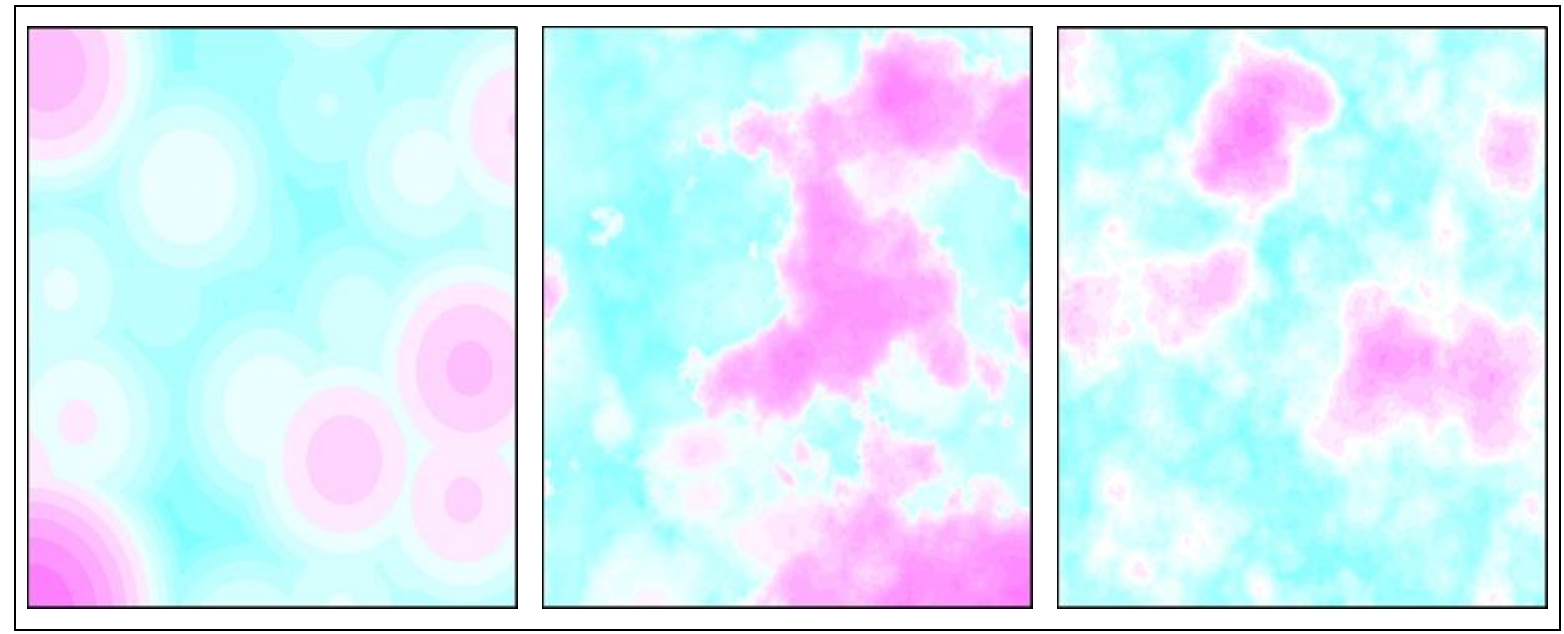

Max-stable processes are fit using their bivariate distributions and pairwise likelihood estimation for reasons of computational practicality (Davison and Gholamrezaee 2011; Ribatet 2013; Ribatet et al. 2015; Ribatet 2017). Model selection among competing max-stable processes can be based on the composite likelihood information criterion, an adaption of the Takeuchi Information Criterion (TIC) (Takeuchi 1976), due to application of the composite pairwise likelihood formulation (Ribatet et al. 2015; Ribatet 2017). However, the use of qualitative evaluations of model fit are also recommended (Ribatet 2017; Ribatet 2015).

The extremal coefficient is a useful measure for summarizing dependence among extreme data (Davison and Gholamrezaee 2011; Ribatet et al. 2015; 
Ribatet 2017). From multivariate extremes, for a max-stable random vector with fixed unit Fréchet margins $Z=\left(Z_{1}, \ldots, Z_{k}\right), k \geq 2, \operatorname{Pr}(Z \leq z)=$ $\exp \left(-V\left(z_{1}, \ldots, z_{k}\right)\right)$ for all $z=\left(z_{1}, \ldots, z_{k}\right) \in(0, \infty)^{k}$ where $V$, the exponent function, satisfies $V\left(\lambda z_{1}, \ldots, \lambda z_{k}\right)=\lambda^{-1} V\left(z_{1}, \ldots, z_{k}\right) \forall \lambda>$ $0, V\left(\infty, \ldots, \infty, z_{d}, \infty, \ldots, \infty\right)=\frac{1}{z_{d}}$ for each $d \in(1, k)$, with two bounding cases; viz., at independence, $V\left(z_{1}, \ldots, z_{k}\right)=\frac{1}{z_{1}}+\cdots+\frac{1}{z_{k}}$, and at dependence, $V\left(z_{1}, \ldots, z_{k}\right)=\min \left(\frac{1}{z_{1}}, \ldots, \frac{1}{z_{k}}\right)$ (Ribatet 2017; Davison et al. 2012; Davison and Gholamrezaee 2011). Hence, $\forall z>0$, the $\left(Z_{1} \leq z, \ldots, Z_{k} \leq z\right)=$ $\exp (-V(z, \ldots, z))=\exp \left(\frac{-V(1, \ldots, 1)}{z}\right)=\exp \left(\frac{-\theta}{z}\right)$, where $\theta$, the extremal coefficient, is a quantity independent of the level of $z$ and a summary measure of the degree of dependence among the extreme data. The extremal coefficient varies between one, when the observations are fully dependent, and $k$, when they are independent (Ribatet 2017; Davison et al. 2012; Davison and Gholamrezaee, 2011). Results from the spectral representation of a max-stable process combined with the understanding that observations of $\left\{Z(x): x \in X \subset \mathbb{R}^{d}, d \geq 1\right\}$ are at discrete locations in $X$ yield $\theta=\mathbb{E}\left\{\max _{j=1, \ldots, k} Y\left(x_{j}\right)\right\}$ (Ribatet et al. 2015; Ribatet 2017; Davison and Gholamrezaee 2011). Hence, the extremal coefficient can be approximated from simulation (Davison and Gholamrezaee 2011). However, for spatial extremes, the extremal coefficient function is a convenient summary measure of dependence among the extreme data (Schlather and Tawn 2003). In the bivariate case, assuming isotropy, the extremal coefficient is a function of the Euclidean distance, $h$, between any two observation sites and the $\operatorname{Pr}(Z(x) \leq z, Z(x+h) \leq z)=\exp (-V(z, z))=\exp \left(\frac{-V(1,1)}{z}\right)=$ $\exp \left(\frac{-\theta(h)}{z}\right)=\mathbb{E}[\max (Y(x), Y(x+h))]$ with $\theta(h) \in[1,2]$. There exists a unique straightforward mapping between the extremal coefficient function, $\theta(h)$, and the F-madogram (Cooley et al. 2006), a well-defined measure of dependence among extreme data. Moreover, there exist empirical estimators for the F-madogram (Ribatet et al. 2015; Cooley et al. 2012). In summary, inspection of plots of the extremal coefficient function for a model and its data is a recommended qualitative evaluation of a fitted simple max-stable model. Using its bivariate distribution and properties of correlation functions for isotropic fields it can be shown that the Schlather process is unable to model spatial independence (Davison and Gholamrezaee 2011). This is the previously mentioned limitation of the Schlather process and the reason it produced larger areas with the largest values in Figures 1 and 2 when compared with the more flexible extremal-t and Brown-Resnick models. 
The discussion so far has focused on the description, fitting, selection, and qualitative evaluation of max-stable processes with fixed unit Fréchet margins. Assuming fixed margins is not realistic for most practical applications. As previously mentioned, the marginal distributions of a max-stable process are GEV distributed, possibly varying by location. The pairwise likelihood used to fit a simple max-stable process can be readily adapted to accommodate the simultaneous estimation of trend surface and dependence parameters (Ribatet et al. 2015; Ribatet 2017; Ribatet 2015). Trend surfaces are functions defined on $X$ which use covariates to model the spatial variation of the location, $\mu(x)$, scale, $\sigma(x)$, and shape, $\xi(x)$, parameters of the known GEV marginal distributions. For example, linear trend surfaces are of the form $\mu(x)=\eta_{\mu, 0}+\eta_{\mu, 1} \operatorname{cov}_{\mu, 1}+\cdots+\eta_{\mu, n_{\mu}} \operatorname{cov}_{\mu, n_{\mu}}$, $\sigma(x)=\eta_{\sigma, 0}+\eta_{\sigma, 1} \operatorname{cov}_{\sigma, 1}+\cdots+\eta_{\sigma, n_{\mu \sigma}} \operatorname{cov}_{\sigma, n_{\sigma}}, \xi(x)=\eta_{\xi, 0}+\eta_{\xi, 1} \operatorname{cov}_{\xi, 1}+\cdots+$ $\eta_{\xi, n_{\xi}} \operatorname{cov}_{\xi, n_{\xi}}$, where $\eta_{, i}$ and $\operatorname{cov}_{\cdot, i}$ are the parameters and covariates of the linear trend surface for $\mu(x), \sigma(x)$, and $\xi(x)$, respectively. Potential covariates include, for example, gridded physiographic (e.g., such as $\mathrm{x}$ location, y-location, elevation, slope, aspect, curvature) and climatological (e.g., such as mean annual/monthly temperature, precipitation, wind, solar radiation) data. Ribatet (2017) underscored the importance of correctly modeling the spatial variation of the marginal parameters by carefully "building relevant trend surfaces including any relevant covariable." Poor characterizations for $\mu(x), \sigma(x)$, and $\xi(x)$ complicates the dependence parameterization (Blanchet 2009; Ribatet 2017). In this study, linear trend surfaces for the marginal parameters were effectively and efficiently developed by applying previously mentioned theory from spatial extremes and recent advances for regularizing general linear models (Friedman et al. 2010; Tibshirani et al. 2010; Simon et al. 2011; Tibshirani et al. 2012).

Regularization of the linear trend surface for $\mu(x), \sigma(x)$, and $\xi(x)$ is needed given the number of available and presumed relevant gridded covariate datasets and acknowledgment that there are $2^{n}$. possible models that involve subsets of $n$. predictors where $n_{.}=n_{\mu}, n_{\sigma}$, or $n_{\xi}$ (Gareth et al. 2013). If $n .=10 / 100$, then there are $1,024 / 1.267651 \mathrm{e}+30$ possible models to be considered for a given marginal parameter trend surface. Zou and Hastie (2005) introduced the elastic-net penalty as a compromise between ridge (Hoerl and Kennard 1970; Tikhonov 1943) and lasso (Tibshirani 1996) regression. Given observations $y_{i}, i=1, \ldots, n$, an $n \times m$ matrix of normalized covariates $X$, and an assumed linear model $y_{i}=\eta_{0}+\eta_{1} x_{i, 1}+$ $\cdots+\eta_{m} x_{i, m}$, the elastic net minimizes $\frac{1}{2 n} \sum_{i=1}^{n}\left(y_{i}-\eta_{0}-\eta x_{i}^{T}\right)^{2}+$ 
$\lambda \sum_{j=1}^{m}\left[\frac{1}{2}(1-\alpha) \eta_{j}^{2}+\alpha\left|\eta_{j}\right|\right]$, where $\lambda$ is a non-negative regularization parameter that is tuned to weight the overall strength of the penalty and $\alpha \in$ $[0,1]$ is specified to control the penalty term to vary from ridge regression at $\alpha=0$ to lasso regression at $\alpha=1$ (Friedman et al. 2010). Ridge regression yields smooth solutions that include all the predictors whereas application of lasso regression results in automatic variable selection (i.e., sparse, much more easily interpretable solutions [Gareth et al. 2013]). The elastic net mixes the two methods. As $\alpha$ increases from o to 1 for a fixed $\lambda$, the number of zero-valued $\eta_{j}$ increases from o to the sparsity of the lasso (Friedman et al., 2010). In this study, variable selection was preferred, and $\alpha$ was specified close to 1 for numerical stability (Friedman et al. 2010). Cross validation was applied to ensure that the minimizing value for $\lambda$ was properly located for each elastic net application.

Independently derived elastic net application results for $\mu(x), \sigma(x)$, and $\xi(x)$ guided subsequent spatial GEV model formulation and evaluation. The log-likelihood of the spatial GEV model, which assumes independence among the sample observation sites, is given by $l\left(\eta_{\mu}, \eta_{\sigma}, \eta_{\xi}\right)=$ $\sum_{i=1}^{n s i t e} \sum_{j=1}^{n o b s}\left\{-\log \sigma_{i}-\left(1+\xi_{i} \frac{y_{i, j}-\mu_{i}}{\sigma_{i}}\right)^{-1 / \xi_{i}}-\left(1+\frac{1}{\xi_{i}}\right) \log \left(1+\xi_{i} \frac{y_{i, j}-\mu_{i}}{\sigma_{i}}\right)\right\}$, where $\mu_{i}, \sigma_{i}$, and $\xi_{i}$ are the GEV parameters for the $i$-th site and $y_{i, j}$ is the $j$-th observation for the $i$-th site (Ribatet 2009). For $\mu(x), \sigma(x)$, and $\xi(x)$, predictors associated with a set of elastic-net derived linear trend surface models, ranging in dimension from 1 to the dimension of the trend surface at the minimizing $\lambda$, were used to define and evaluate a series of spatial GEV models. For example, if 10, 5, and 3 elastic-net directed trend surface models were extracted from applications of elastic net for $\mu(x), \sigma(x)$, and $\xi(x)$, respectively, then 150 unique spatial GEV models were defined, fitted, and evaluated. Spatial GEV model selection and evaluation was based on information criterion scores and comparisons of the spatial GEV model parameter estimates with their at-site counterparts (Blanchet 2009; Ribatet 2009).

The max-stable process deployment herein was a two-step procedure involving trend surface and simple max-stable process model selection, with each step assuming independence among the extremes and fixed margins, respectively. The results from these two separate steps were subsequently combined to build a general max-stable process wherein the trend surface and dependence parameters were simultaneously fitted. Thereafter, pointwise and areal-based return levels can be computed for any 
arbitrary area within the model domain. Areal-based exceedances are obtained by simulating multiple independent copies of the fitted max-stable process (Ribatet 2009; Ribatet 2013; Ribatet 2015; Ribatet 2017). Schlather (2002) introduced the basis for simulating an independent realization of a simple max-stable process with on only a finite number of replicates. 


\section{Snow Water Equivalent (SWE) Observations}

SWE time-series data were collected from 112 observation sites across four distinct surface networks as shown in Figure 3. The four SWE observation networks included the Hubbard Brook Experimental Forest (HBEF) maintained by the US Forest Service (http://data.hubbardbrook.org/data/dataset.php?id=28), Soil Climate Analysis Network (SCAN) data managed by the Natural Resources Conversation Service (https://www.wcc.nrcs.usda.gov/scan/), snow sampling sites maintained by the New Hampshire Department of Environmental Services (NHDES) (https://www4.des.state.nh.us/Rti_home/snow_sampling_stations.asp), and snow survey data from the New England District of the US Army Corps of Engineers (USACE)

(https://reservoircontrol.usace.army.mil/NE/pls/cwmsweb/cwms_web.cwmsweb.cwmsindex). Table 1 summarizes the number of SWE observation sites and annual maxima associated with each network. The number of processed annual maxima (AM) for each observation site for the period 1950-2017 varied from 18 to 68 and averaged 25.4 across all 112 sites. The average was influenced by the 2000-2017 period of record for the USACE-observed SWE time-series datasets. The SWE AM were observed early to mid-March on average. Figure 4 is a symbol plot of the mean of the SWE AM data, in inches, for each observation site, and it underscores the spatial variation of the data within and surrounding the upper Connecticut River Basin (CRB). 
Figure 3. SWE observation site locations overlain on a digital elevation model (DEM) (SWE networks: ==HBEF; ==SCAN; •=NHDES; ==USACE; DEM data source: PRISM 30 year normal (Norm81m dataset) (http://www.prism.oregonstate.edu/normals/).

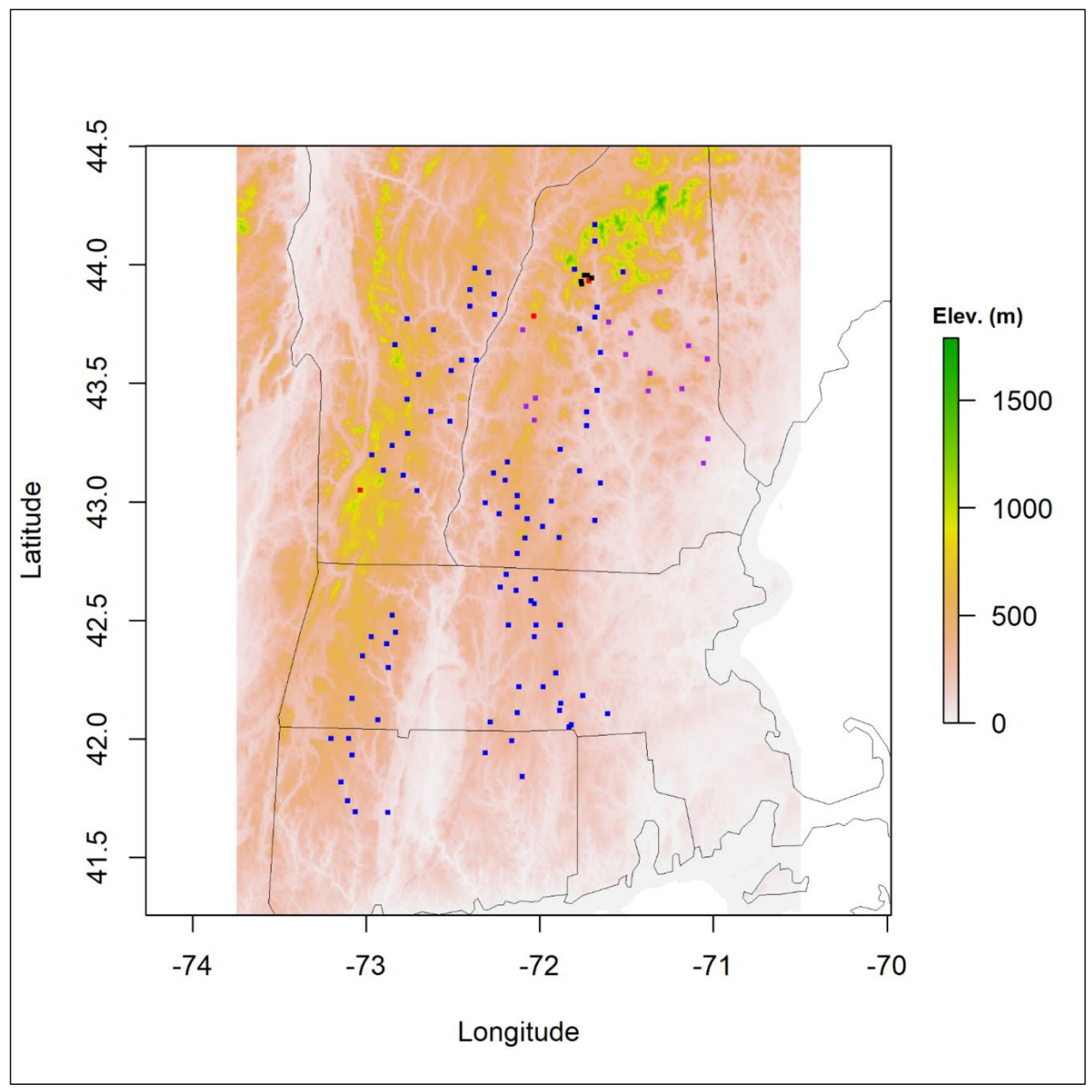

Table 1. Number of observation sites and annual maxima associated with each SWE network.

\begin{tabular}{|c|c|c|c|}
\hline SWE Network & $\begin{array}{c}\text { Number of } \\
\text { Observation Sites }\end{array}$ & Period of Record & $\begin{array}{c}\text { Number of } \\
\text { Annual Maxima }\end{array}$ \\
\hline HBEF & 5 & $1955-2015$ & 305 \\
\hline SCAN & 3 & $2000-2017$ & 54 \\
\hline NHDES & 15 & $1950-2017$ & 889 \\
\hline USACE & 89 & $2000-2017$ & 1602 \\
\hline
\end{tabular}


Figure 4. Mean of the SWE annual maxima (inches) for each SWE observation location overlain on a digital elevation model (DEM) (DEM data source: PRISM 30 year normal (Norm81m dataset) (http://www.prism.oregonstate.edu/normals/).

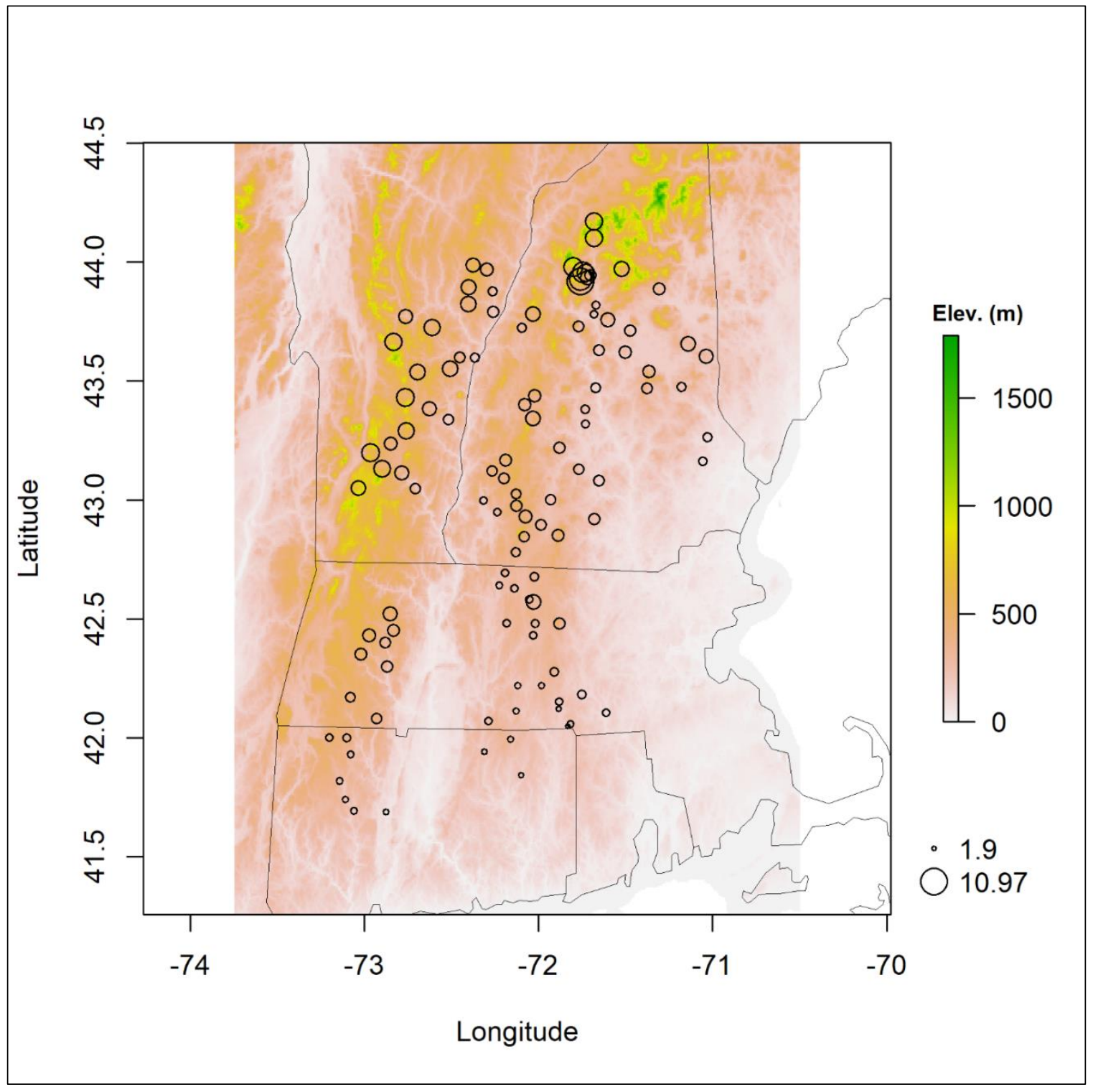




\section{Covariate Datasets}

Gridded datasets of likely relevant physiographic and climatological covariates were collected and processed to support trend surface modeling of the SWE AM for the latitude and longitude box defined by $(41.25,44.5) \times$ $(-73.75,-70.5)$ (Sexstone and Fassnacht 2014). Table 2 is a summary listing of the collected and processed gridded covariate datasets and their sources.

Table 2. Summary listing of the 135 collected and processed base covariates. Monthly, annual, and seasonal (average of November - April monthlies) products were collected and processed for the climatology datasets (PRECIP, TMEAN, TMAX, TMIN, TDMEAN, DLWRF, DSWRF, EWIND, NWIND).

\begin{tabular}{|c|c|c|}
\hline \multicolumn{3}{|c|}{$\begin{array}{l}\text { Source: PRISM } 30 \text { year normal (Norm81m dataset) (http://www.prism.oregonstate.edu/normals/) } \\
\text { (http://www.prism.oregonstate.edu/documents/PRISM_datasets.pdf) }\end{array}$} \\
\hline Covariates & Description & Resolution \\
\hline $\mathrm{X}, \mathrm{Y}, \mathrm{Z}$ (from DEM) & Coordinates and elevation for each raster grid cell & \multirow[b]{6}{*}{$30 \operatorname{arcsec}{ }^{*}$} \\
\hline PRECIP & Total precipitation & \\
\hline TMAX & Maximum temperature & \\
\hline TMIN & Minimum temperature & \\
\hline TMEAN & $0.5 *($ TMAX+TMIN $)$ & \\
\hline TDMEAN & Mean dew point temperature & \\
\hline \multicolumn{3}{|c|}{$\begin{array}{l}\text { Source: Geographic Resources Analysis Support System, Geographic Information System function } \\
\text { r.slope.aspect (https://grass.osgeo.org/grass70/manuals/r.slope.aspect.html) }\end{array}$} \\
\hline SLOPE & Slope & \\
\hline ASPECT & Aspect & \\
\hline $\begin{array}{l}\text { PROFILE } \\
\text { CURVATURE }\end{array}$ & Profile curvature & \\
\hline $\begin{array}{l}\text { TANGENTIAL } \\
\text { CURVATURE }\end{array}$ & Tangential curvature & 30 arc sec \\
\hline \multicolumn{3}{|c|}{ Source: Global 2010 Tree Cover $\left(30\right.$ m) ${ }^{\dagger}$ (https://glad.umd.edu/dataset/global-2010-tree-cover-30-m) } \\
\hline TREE COVER & $\begin{array}{l}\text { Percent maximum (peak of growing season) tree canopy cover (circa } \\
\text { 2010) }\end{array}$ & 1 arc sec \\
\hline
\end{tabular}

* For a full list of the spelled-out forms of the units of measure used in this document, please refer to US Government Publishing Office Style Manual, 31st ed. (Washington, DC: US Government Publishing Office, 2016), 248-52, https://www.govinfo.gov/content/pkg/GPO-STYLEMANUAL-2016/pdf/GPOSTYLEMANUAL-2016.pdf.

† For a full list of the unit conversions used in this document, please refer to US Government Publishing Office Style Manual, 31st ed. (Washington, DC: US Government Publishing Office, 2016), 345-7, https://www.govinfo.gov/content/pkg/GPO-STYLEMANUAL-2016/pdf/GPO-STYLEMANUAL-2016.pdf. 
Source: National Aeronautics and Space Administration, North American Land Data Assimilation System (https://hydro1.gesdisc.eosdis.nasa.gov/data/NLDAS/README.NLDAS2.pdf)

\begin{tabular}{|l|l|l|}
\hline DLWRF & Downward longwave radiation flux & \\
\cline { 1 - 2 } DSWRF & Downward shortwave radiation flux & $.125 \mathrm{deg}$ \\
\hline
\end{tabular}

Source: National Oceanic and Atmospheric Administration National Centers for Environmental Prediction Climate Forecast System Reanalysis dataset (https://app.climateengine.org/)

\begin{tabular}{|l|l|l|}
\hline EWIND & Eastward wind component & \\
\hline NWIND & Northward wind component & $.2 \mathrm{deg}$ \\
\hline Source: Historical climate data for North America (https://sites.ualberta.ca/ ahamann/data/climatena.html) \\
\hline PPAS & Percent precipitation as snow & $1 \mathrm{~km}$ \\
\hline
\end{tabular}




\section{Analysis}

As previously mentioned, the max-stable model development process involved two key steps:

1. characterizing the spatial/spatiotemporal variation of the extreme data (i.e., trend surface model selection)

2. accounting for the inter-site dependence among the extreme data (i.e., simple max-stable model selection).

This section succinctly highlights salient features associated with these two steps necessary for the deployment of a max-stable process model of SWE $\mathrm{AM}$ for the upper CRB.

\subsection{Trend surface modeling}

Figure 5 depicts the spatial distribution of at-site estimates of GEV model parameters for each SWE observation location, underscoring that a distinct unique trend surface likely exists for $\mu(x), \sigma(x)$, and $\xi(x)$, respectively. The 135 base spatial covariates (extracted at the 30 arc sec resolution), and their squares, constitute the entire set of 270 covariables considered to build each trend surface. Figure 6 includes summary plots associated with three independent elastic net applications to build trend surfaces for $\mu(x), \sigma(x)$, and $\xi(x)$, respectively. Each respective elastic net application was performed using the R software package glmnet (Friedman et al. 2010), employing $k$-fold cross validation with $k=37$, and with $\alpha$ specified equal to 0.95. For each plot in Figure 6, the $x$-axis is the natural logarithm of $\lambda$, the non-negative regularization parameter that is tuned to weight the overall strength of the penalty, the $y$-axis is the mean squared error (MSE), the top of the plot indicates the number of non-zero covariates as $\lambda$ varies, the red curve and its error bars is the crossvalidation derived MSE including its one standard error lower and upper bounds, and the two dotted vertical lines indicate the locations of the cross-validation identified $\lambda$ value that minimizes the MSE and the defined best regularizing model (Hastie and Qian 2016). The best regularizing model is defined to be at the largest $\lambda$ value within one standard error of the minimizing MSE (Friedman et al. 2010; Hastie et al. 2009). Figure 7 contains additional summary plots that are simply a rearrangement of the data depicted in the plots from Figure 6. The plots in Figure 7 clearly emphasize the tradeoff between mean MSE fit and model complexity for 
each of the cross-validated elastic net derived independent trend surfaces for $\mu(x), \sigma(x)$, and $\xi(x)$, respectively.

Several spatial GEV models were subsequently defined, fitted, and evaluated based on the cross-validation supervised elastic net applications, which identified optimal independent trend surfaces for $\mu(x), \sigma(x)$, and $\xi(x)$, respectively. Besides the minimizing and best regularizing models, additional trend surface models for $\mu(x), \sigma(x)$, and $\xi(x)$ were extracted from each of the curves shown in Figure 6. Tables $3-5$ summarize the 10, 7 , and 4 trend surface models that were extracted for $\mu(x), \sigma(x)$, and $\xi(x)$, respectively, for subsequent spatial GEV model definition, fitting, and evaluation. For each independent trend surface, models were extracted with the intent to cover the range $\left[\lambda_{\text {min }}, \lambda_{\text {reg }}\right] \cup\left(\lambda_{\text {reg }}, \lambda_{\text {max }}\right)$ where $\lambda_{\text {min }}, \lambda_{\text {reg }}$, and $\lambda_{\text {max }}$ are the $\lambda$ values corresponding with the minimizing, best regularizing, and intercept only models, respectively. Tables $3-5$ specify for $\mu(x), \sigma(x)$, and $\xi(x)$, respectively, the extracted $\lambda$-valued trend surface models and, for each $\lambda$ model, the corresponding cross-validated mean MSE, number of non-zero covariates, and percent deviance explained. A total of $10+10 \cdot 7+10 \cdot 7 \cdot 4=360$ spatial GEV trend surface models were defined, fitted, and evaluated based on the independently derived elastic net trend surface models for $\mu(x), \sigma(x)$, and $\xi(x)$ that are summarized in Tables $3-5$, respectively. The first 10 spatial GEV fits only allowed the GEV location to vary, based on the $\lambda$ models for $\mu(x)$ specified in Table 3. For these first 10 spatial GEV fits, $\sigma(x)$ and $\xi(x)$ are intercept only models. The next $10 \cdot 7$ spatial GEV fits allowed $\mu(x)$ and $\sigma(x)$ to vary as defined in Tables 3 and 4 , respectively, while $\xi(x)$ remained fixed as an intercept only model. The final $10 \cdot 7 \cdot 4$ spatial GEV fits allowed $\mu(x), \sigma(x)$, and $\xi(x)$ to vary as defined in Tables $3-5$, respectively. Figure 8 is a summary of the 360 spatial GEV model evaluations, as measured by their information criterion scores. The spatial GEV model with the lowest information criterion score was defined by the minimizing MSE trend surfaces for $\mu(x)$ and $\sigma(x)$ and effectively the best regularizing model, with two non-zero covariates, for $\xi(x)$. Figure 9 includes scatter plots which compare the spatial GEV model with the lowest information criterion score with its at-site estimates. Computed correlation values associated with the scatter plots shown in Figure 9 are $0.923,0.877$, and 0.581 for $\mu, \sigma$, and $\xi$, respectively. 
Figure 5. Spatial distribution of the at-site extremal model parameters (a) $\mu$, (b) $\sigma$, and (c) $\xi$, respectively.

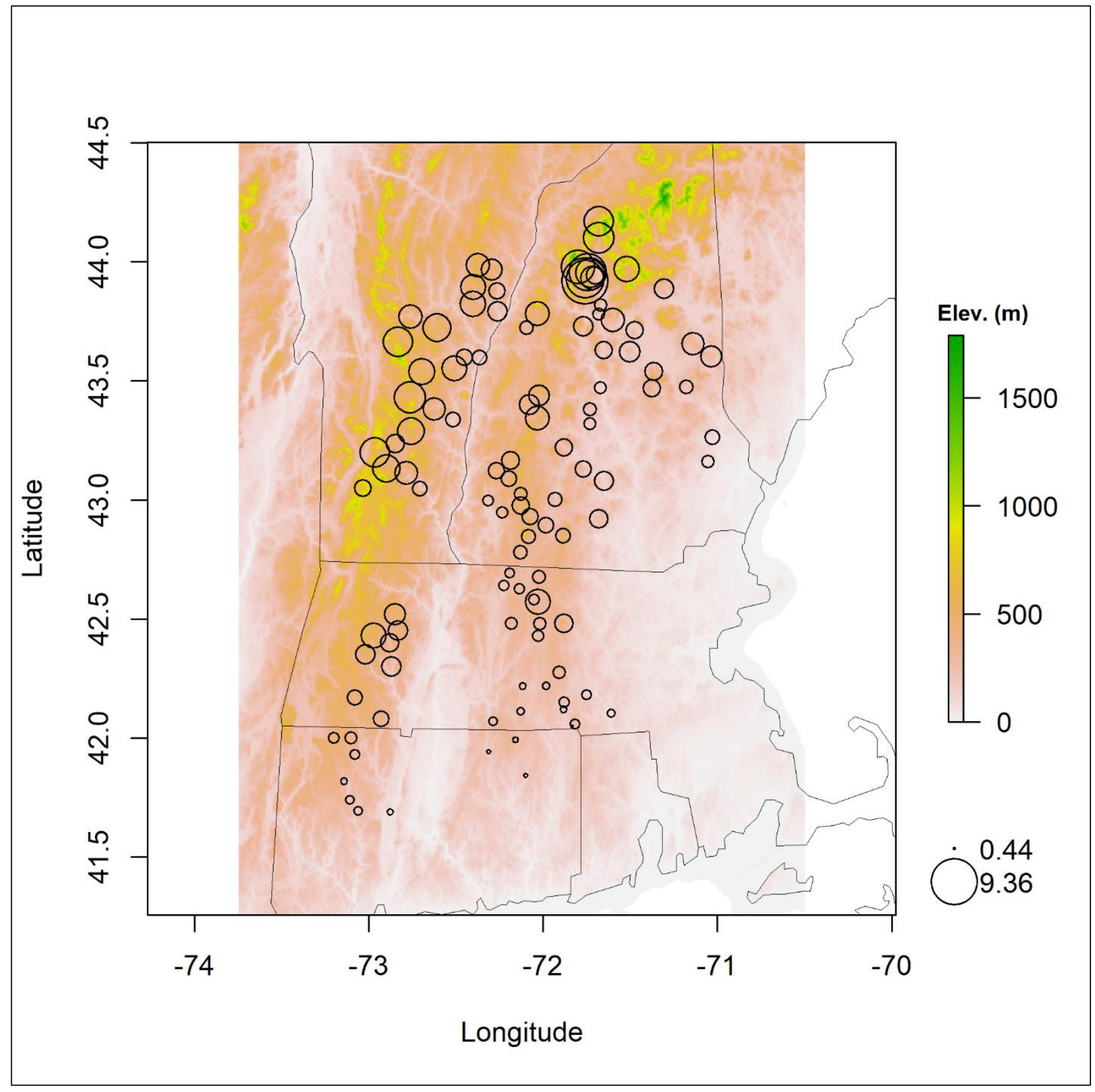

(a) 
Figure 5. Continued.

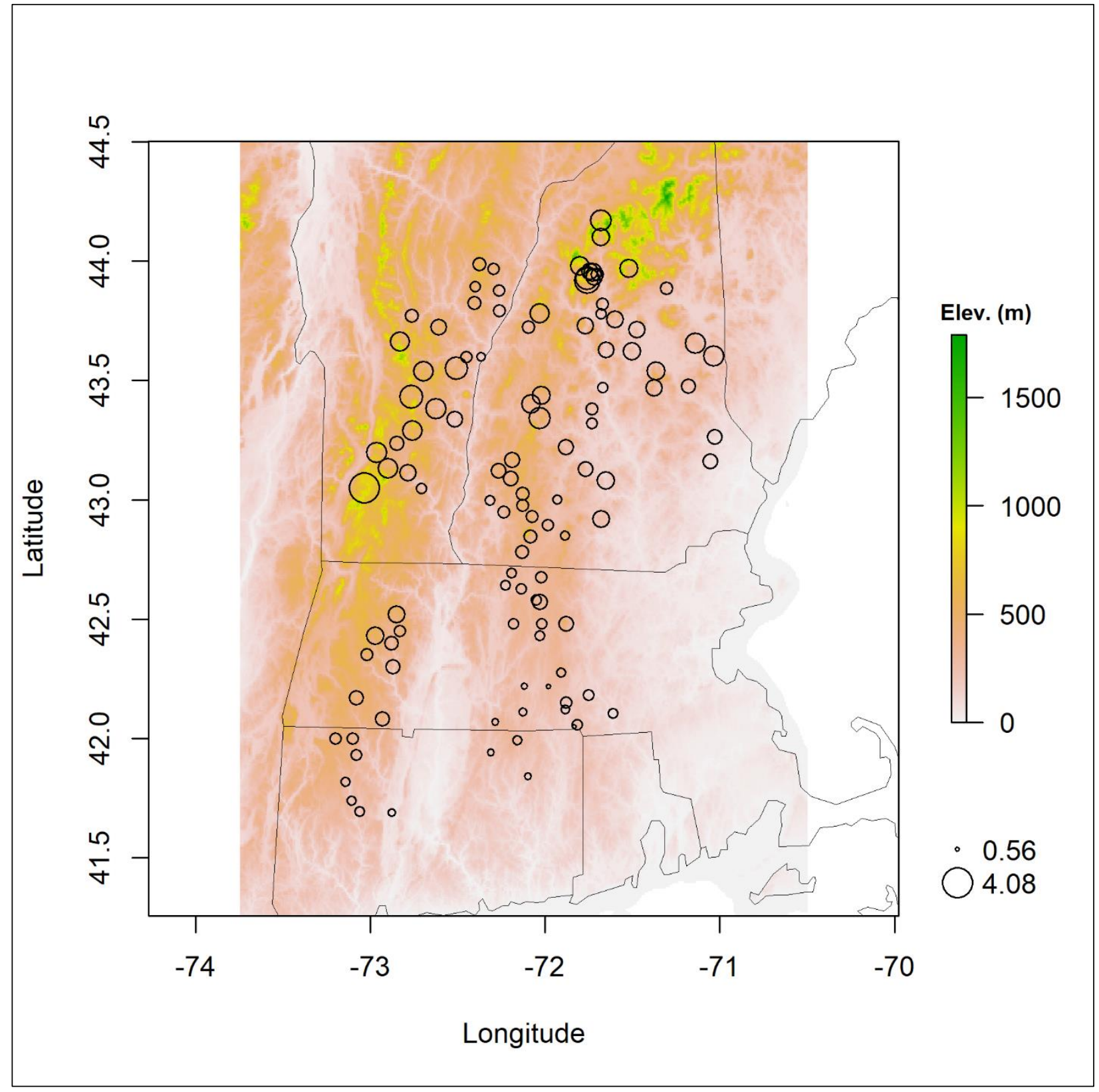

(b) 
Figure 5. Continued.

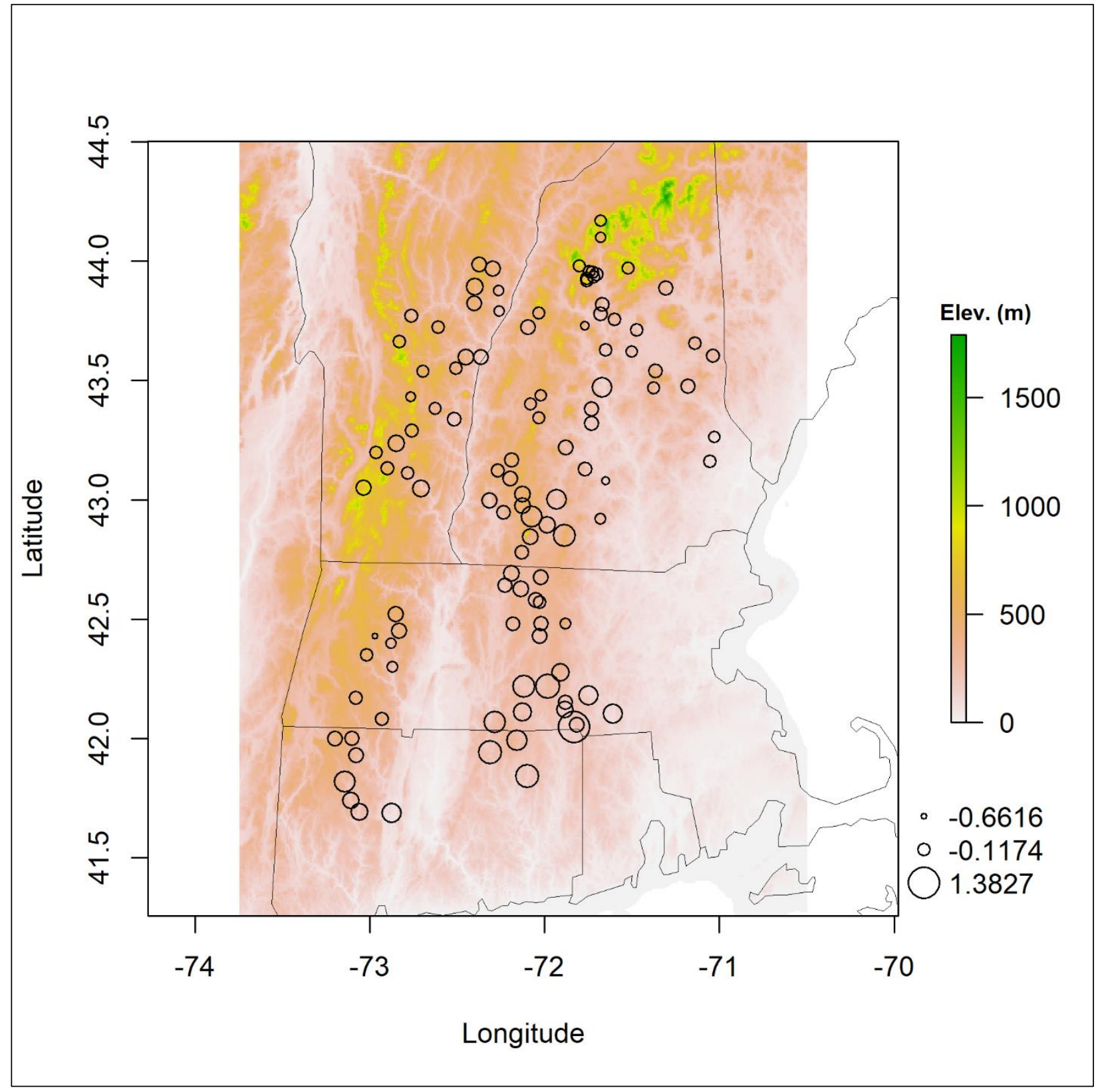

(c) 
Figure 6. Summary plots associated with three independent elastic net applications to build trend surfaces for (a) $\mu(x)$, (b) $\sigma(x)$, and (c) $\xi(x)$, respectively.

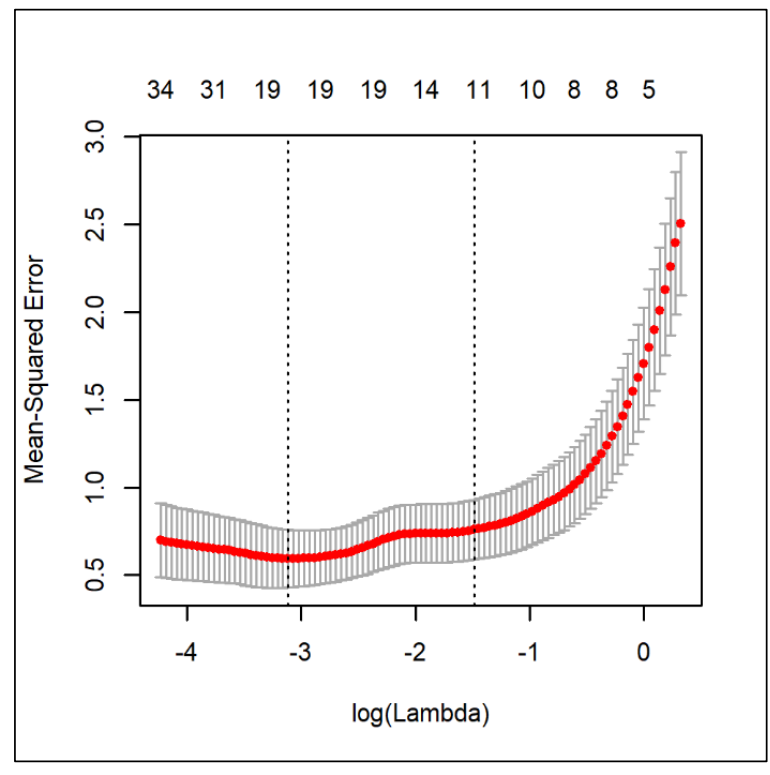

(a)

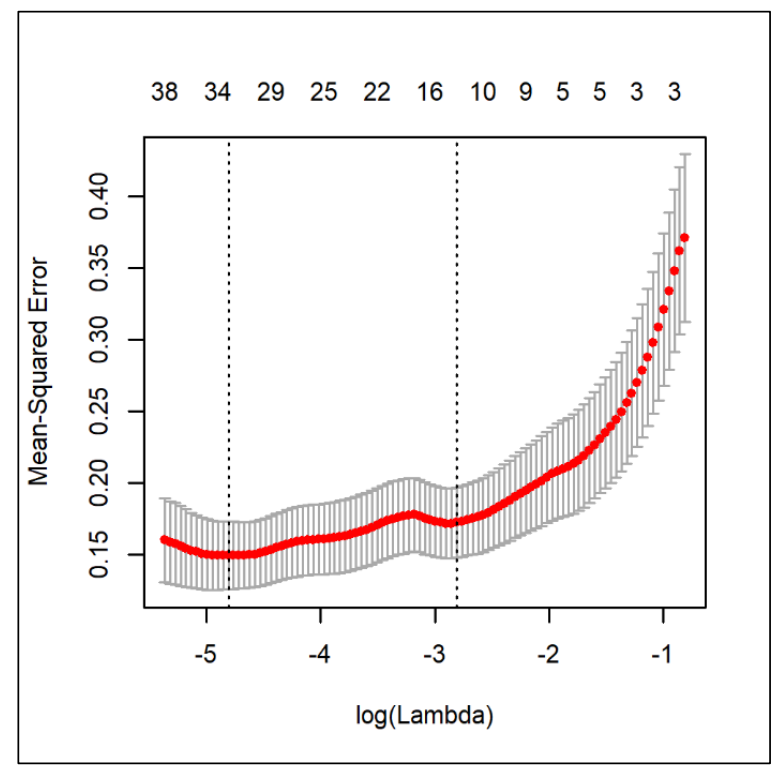

(b)

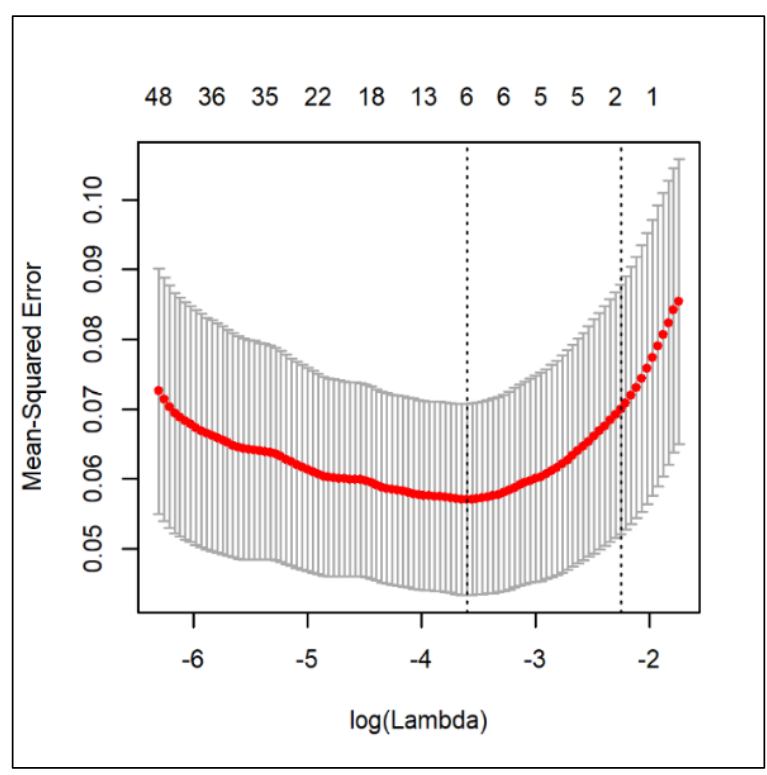

(c) 
Figure 7. Summary plots associated with three independent elastic net applications to build trend surfaces for (a) $\mu(x),(b) \sigma(x)$, and (c) $\xi(x)$, respectively, that underscore the tradeoff between model fit and complexity.

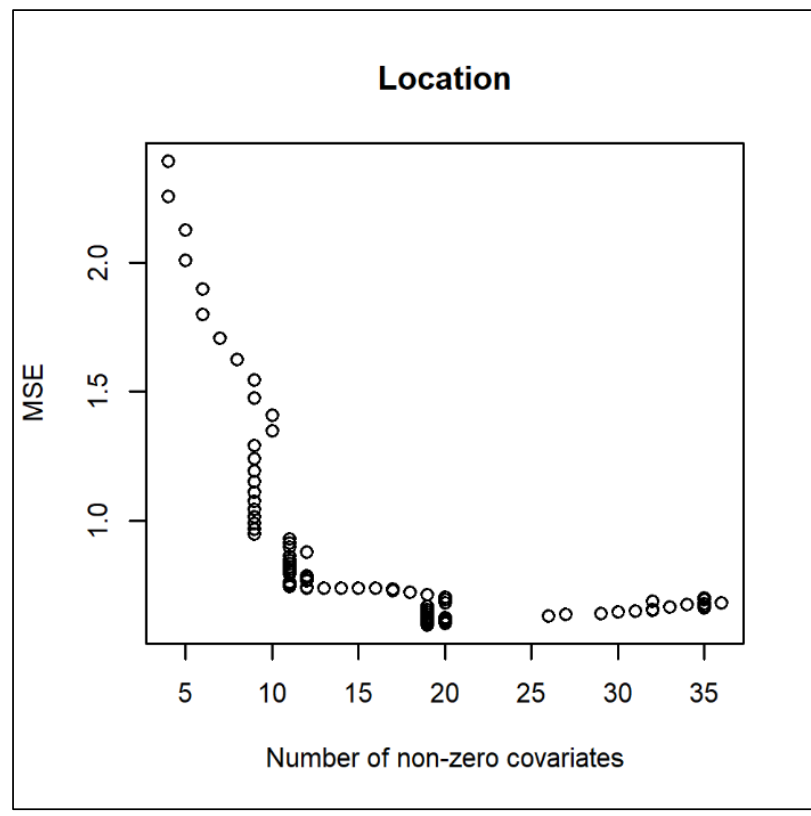

(a)

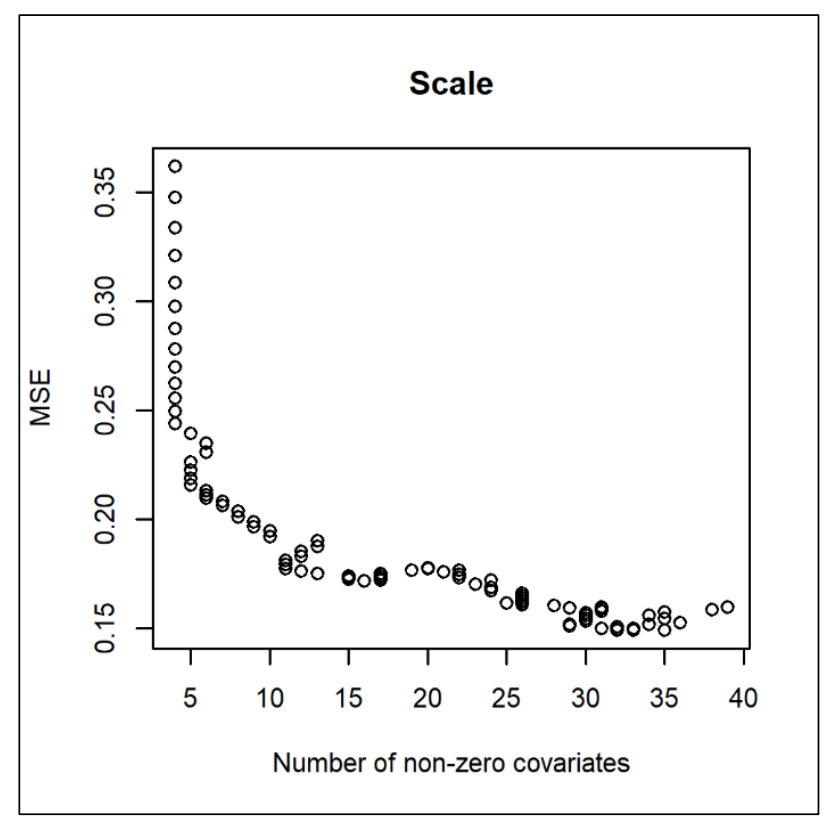

(b)

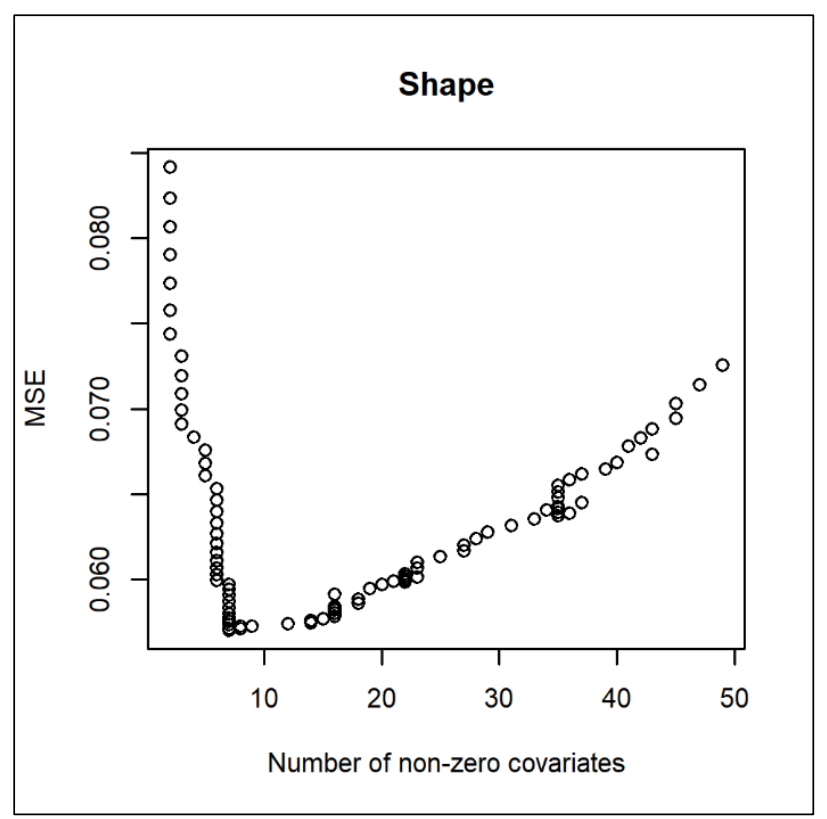

(c) 
Table 3. Summary of elastic net derived trend surfaces for $\boldsymbol{\mu}(\boldsymbol{x})$ extracted for spatial GEV model definition, fitting, and evaluation ( $*=$ best regularizing $\lambda$ model;

$* *=$ minimizing $\lambda$ model).

\begin{tabular}{|c|c|c|c|c|}
\hline Model Index & $\begin{array}{c}\text { Number of } \\
\text { Non-Zero } \\
\text { Covariates }\end{array}$ & Mean MSE & $\%$ Deviance & $\lambda$ \\
\hline 01 & 0 & 2.5034 & 0.00000 & 1.38700 \\
\hline 02 & 3 & 2.2585 & 0.12280 & 1.26400 \\
\hline 03 & 4 & 2.0086 & 0.22560 & 1.15100 \\
\hline 04 & 5 & 1.7984 & 0.31160 & 1.04900 \\
\hline 05 & 8 & 0.9476 & 0.66290 & 0.47570 \\
\hline 06 & $10^{*}$ & 0.7591 & 0.75440 & 0.22600 \\
\hline 07 & 12 & 0.7377 & 0.77210 & 0.16320 \\
\hline 08 & 14 & 0.7383 & 0.77640 & 0.14870 \\
\hline 09 & 16 & 0.7298 & 0.79180 & 0.11780 \\
\hline 10 & $18^{* *}$ & 0.5951 & 0.84960 & 0.04437 \\
\hline
\end{tabular}

Table 4. Summary of elastic net derived trend surfaces for $\sigma(x)$ extracted for spatial GEV model definition, fitting, and evaluation ( $*=$ best regularizing $\lambda$ model; $* *=$ minimizing $\lambda$ model).

\begin{tabular}{|c|c|c|c|c|}
\hline Model Index & $\begin{array}{c}\text { Number of } \\
\text { Non-Zero } \\
\text { Covariates }\end{array}$ & Mean MSE & $\%$ Deviance & $\lambda$ \\
\hline 01 & 3 & 0.2442 & 0.36990 & 0.24330 \\
\hline 02 & 5 & 0.2099 & 0.48720 & 0.15280 \\
\hline 03 & 8 & 0.1968 & 0.54650 & 0.11560 \\
\hline 04 & 10 & 0.1777 & 0.61380 & 0.07605 \\
\hline 05 & $14^{*}$ & 0.1726 & 0.63540 & 0.06027 \\
\hline 06 & 22 & 0.1706 & 0.71260 & 0.02999 \\
\hline 07 & $31^{* *}$ & 0.1495 & 0.81880 & 0.00815 \\
\hline
\end{tabular}


Table 5. Summary of elastic net derived trend surfaces for $\xi(x)$ extracted for spatial GEV model definition, fitting, and evaluation. The minimizing model with a mean MSE value of 0.0571 was at $\lambda=0.0272243$ with 6 non-zero covariates, and the best regularizing model, with a mean MSE equal to 0.07 , was at $\lambda=0.1049096$, with two non-zero covariates.

\begin{tabular}{|c|c|c|c|c|}
\hline Model Index & $\begin{array}{c}\text { Number of Non- } \\
\text { Zero Covariates }\end{array}$ & Mean MSE & $\%$ Deviance & $\boldsymbol{\lambda}$ \\
\hline 1 & 1 & 0.0744 & 0.15500 & 0.12640 \\
\hline 2 & 2 & 0.0691 & 0.22180 & 0.10010 \\
\hline 3 & 4 & 0.0661 & 0.26820 & 0.08314 \\
\hline 4 & 5 & 0.0600 & 0.34270 & 0.04984 \\
\hline
\end{tabular}

Figure 8. Plot of information criterion scores for the defined, fitted, and evaluated spatial GEV models (model naming convention: "Mod" + “ $\xi(x)$ Model Index" + “ $\sigma(x)$ Model Index" + “ $\boldsymbol{\mu}(\boldsymbol{x})$ Model Index").

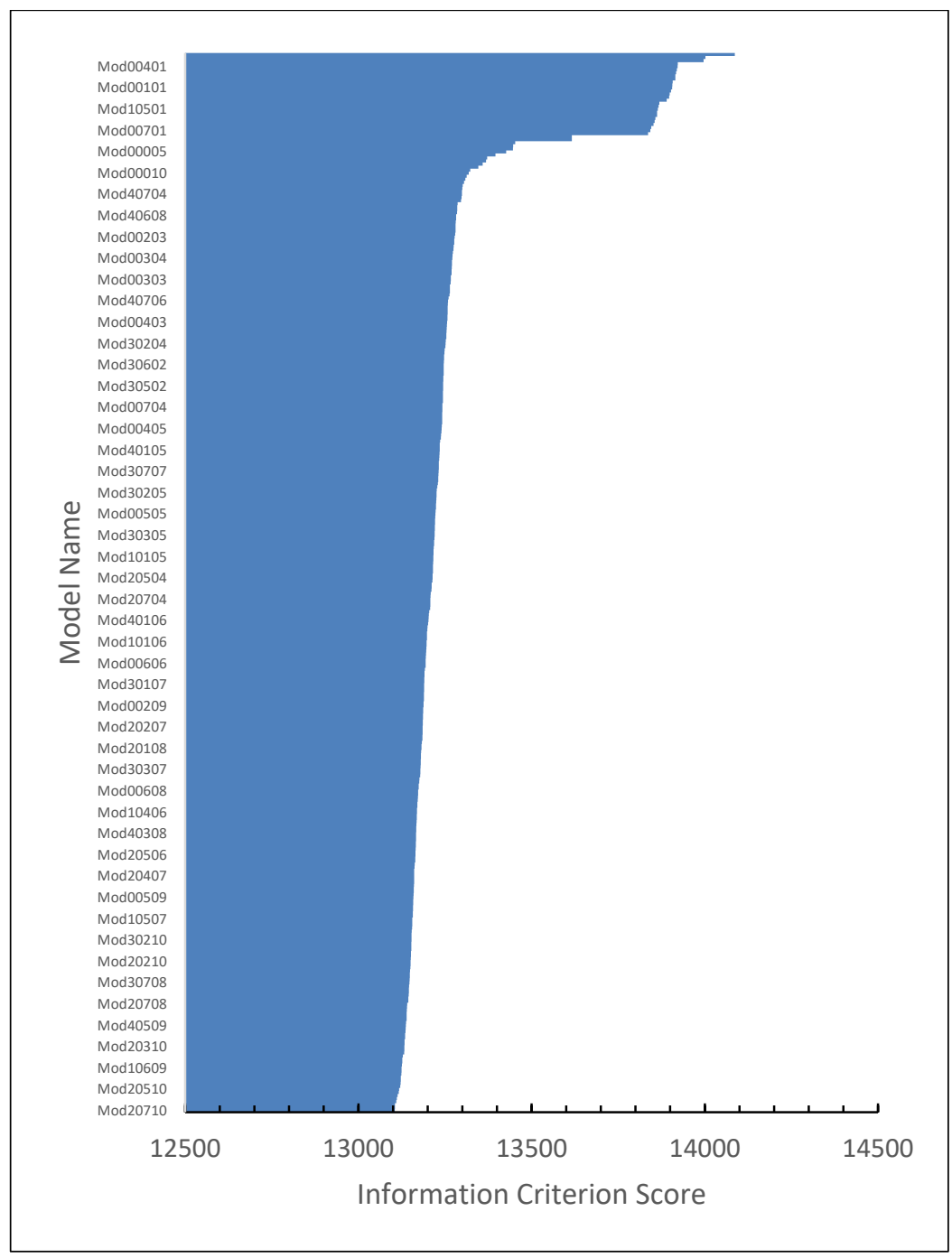


Figure 9. Comparisons of the fitted spatial GEV trend surface models with corresponding at-site estimates ( $\mu=$ GEV location; $\sigma=$ GEV scale; $\xi=$ GEV shape; Model = spatial model; MLE = at-site maximum likelihood estimate).

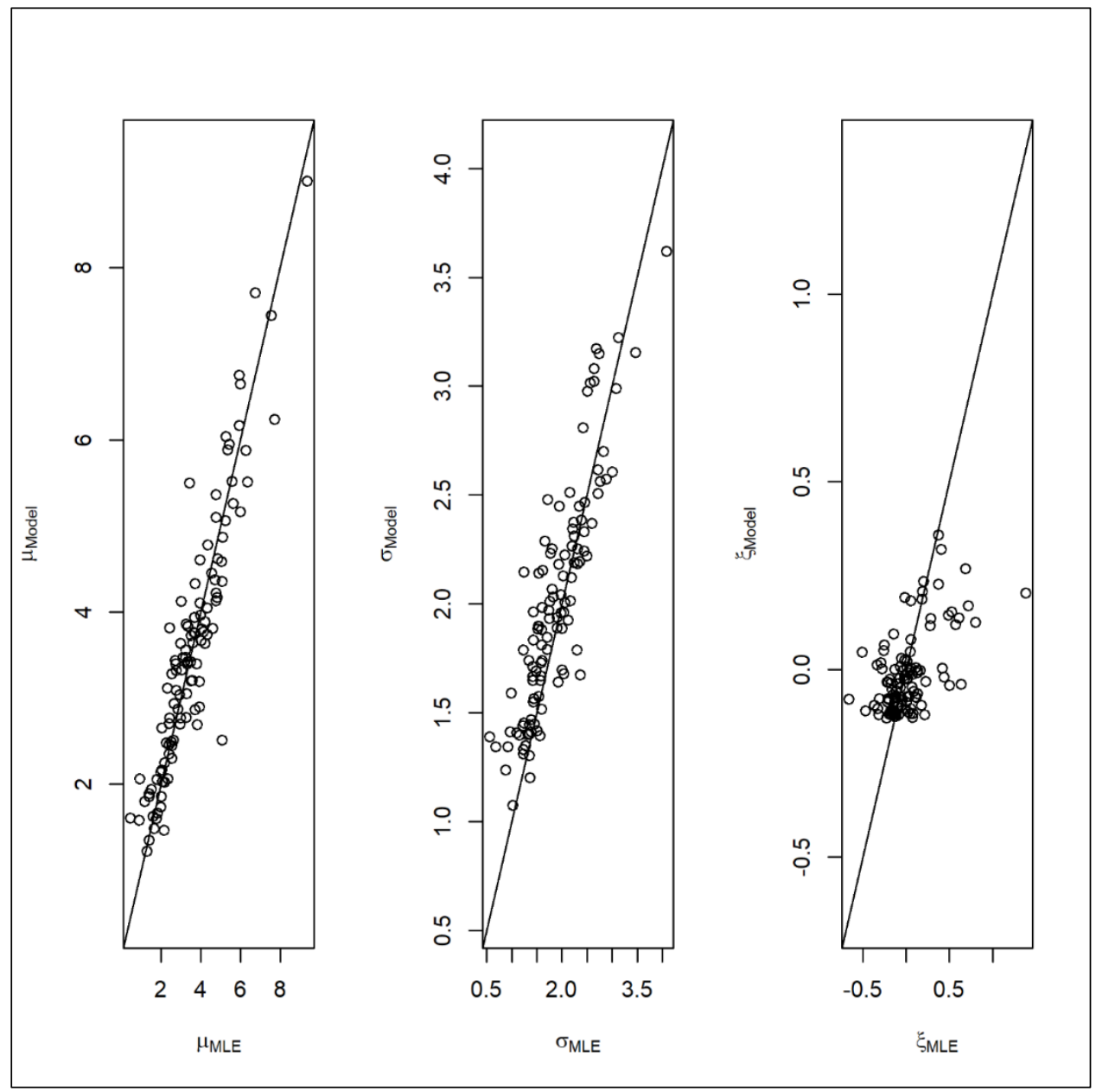

\subsection{Simple max-stable model selection}

The Extremal-t max-stable process was fit to the observed pointwise SWE AM, with each site's data transformed to the unit Fréchet distribution to solely account for the spatial dependence among the data. Five different correlation functions were considered, viz., the Bessel, Cauchy, generalized Cauchy, powered exponential, and Whittle-Matern correlation functions (Ribatet 2018). The Extremal-t process using a Whittle-Matern correlation function was selected based on its lowest information criterion score. Values of 59.6616, 0.1766, and 1.6451 were estimated for its range, smooth, and degrees of freedom parameters, respectively. Figure 10 is a plot of the extremal coefficient function, a succinct measure of spatial dependence as a function of distance, associated with the fitted Extremal-t process together with binned 
pairwise observation estimates. It depicts a non-trivial spatial dependence among the SWE AM in the upper CRB at a normalized distance of 4 , which is approximately 180 miles.

Figure 10. Summary measure of fit for the selected Extremal-t simple max-stable process $(\theta$ is the extremal coefficient function; $h$ is normalized distance).

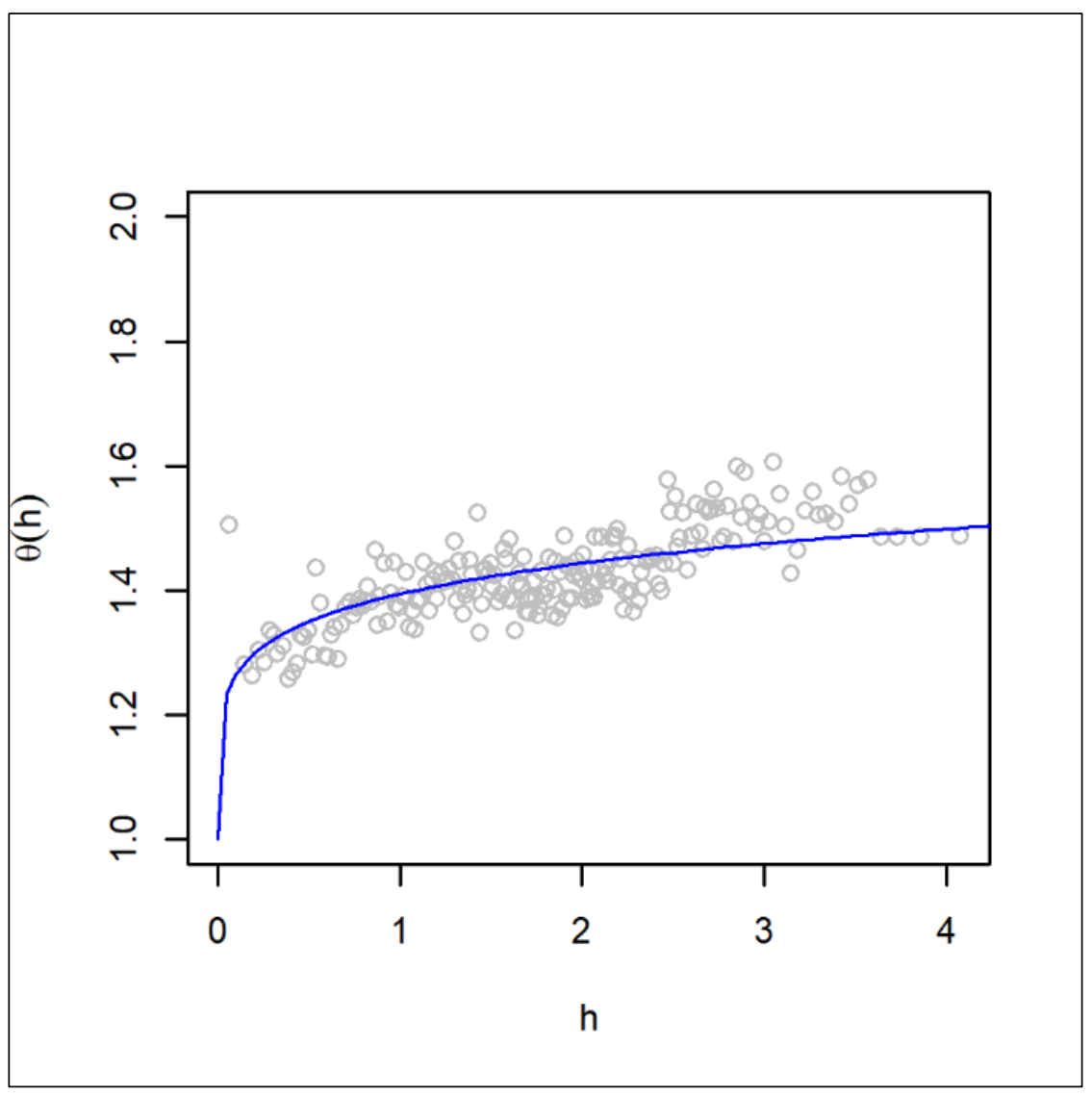

\subsection{General max-stable model development and application}

A general max-stable model was subsequently fit, using constrained optimization, which combined information learned from the separate efforts that focused on trend surface model selection while assuming independence among the sites and simple max-stable process model selection which assumed fixed margins (Ribatet 2017). Figures 11 and 12, extremal coefficient function and scatter plots associated with the fitted general max-stable model that simultaneously estimated the dependence and trend surface parameterizations, agree well with their respective counterparts, which were obtained independently rather than simultaneously, viz., Figures 10 and 9, respectively. The plots in Figure 13 present the fitted trend surfaces for $\mu(x), \sigma(x)$, and $\xi(x)$, and resulting 
pointwise return levels for SWE AM, in inches, at the 100-year return period, respectively. As previously mentioned, with a fitted max-stable process, one can readily compute, via simulation, areal-based exceedances for any arbitrary area (e.g., sub-basin(s), or elevation band(s)) within the model domain. Figure 14 depicts two simulated realizations of the fitted max-stable process for SWE AM, in inches, for the approximately 690-square mile White River Basin located in the upper CRB. Figure 15 is a computed histogram, based on 200 simulations of the fitted process, of mean SWE AM, in inches, for the White River Basin. With simulations from a fitted max-stable process of a hydrometeorological parameter for an area of interest one can readily estimate areal exceedance probabilities for context, understanding, and risk evaluation (e.g., for PFHA, areal precipitation totals, snow totals, probable maximum precipitation estimates, temperatures, sea heights). Table 6 is a summary of exceedance probability estimates, derived using the distribution shown in Figure 15, of the US National Weather Service's National Operational Hydrologic Remote Sensing Center SNOw Data Assimilation System (SNODAS) modeled mean SWE AM for the White River Basin for 2004-2017, inclusive.

Figure 11. Summary diagnostic of fitted general max-stable process model.

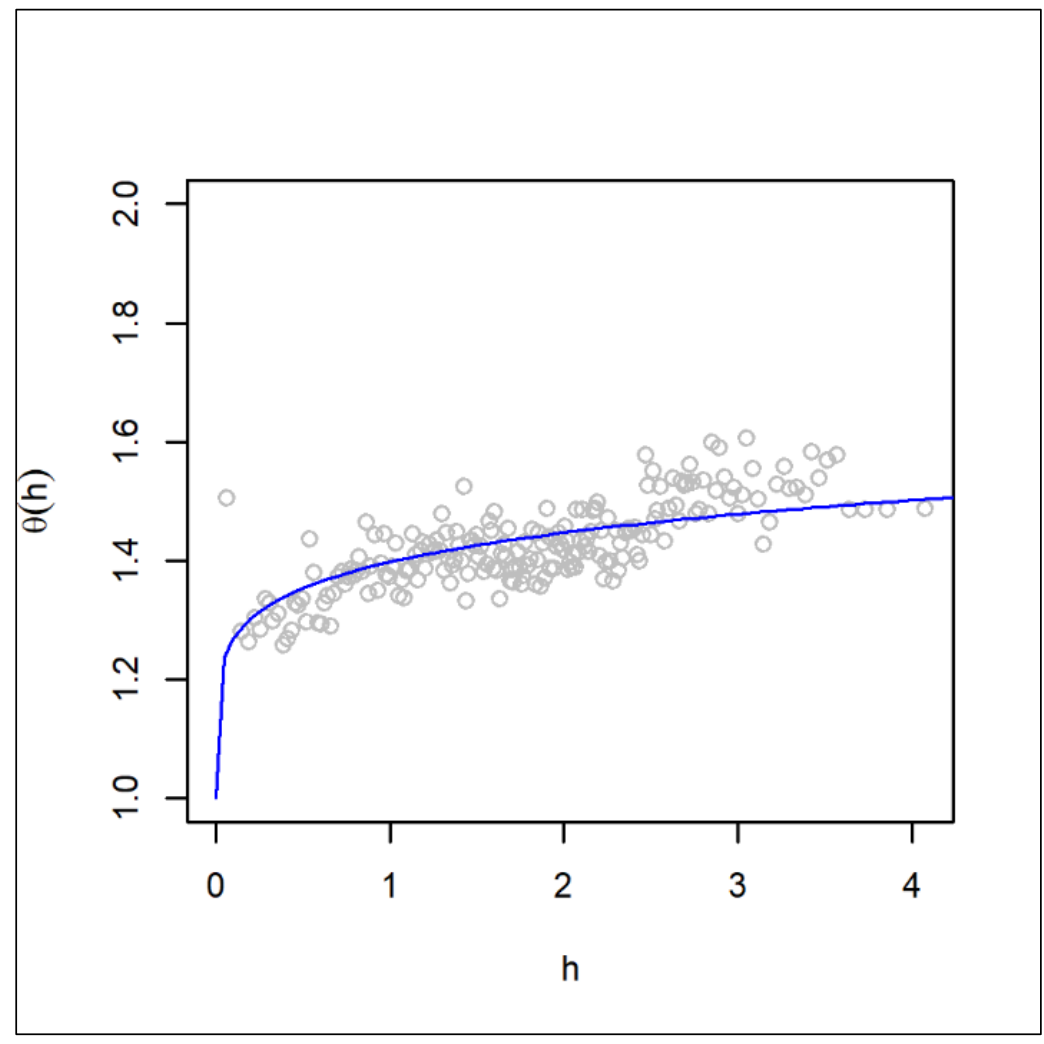


Figure 12. Comparisons of the fitted general max-stable model with corresponding atsite estimates ( $\mu=$ GEV location; $\sigma=$ GEV scale; $\xi=$ GEV shape; Model = general maxstable spatial model; MLE = at-site maximum likelihood estimate).

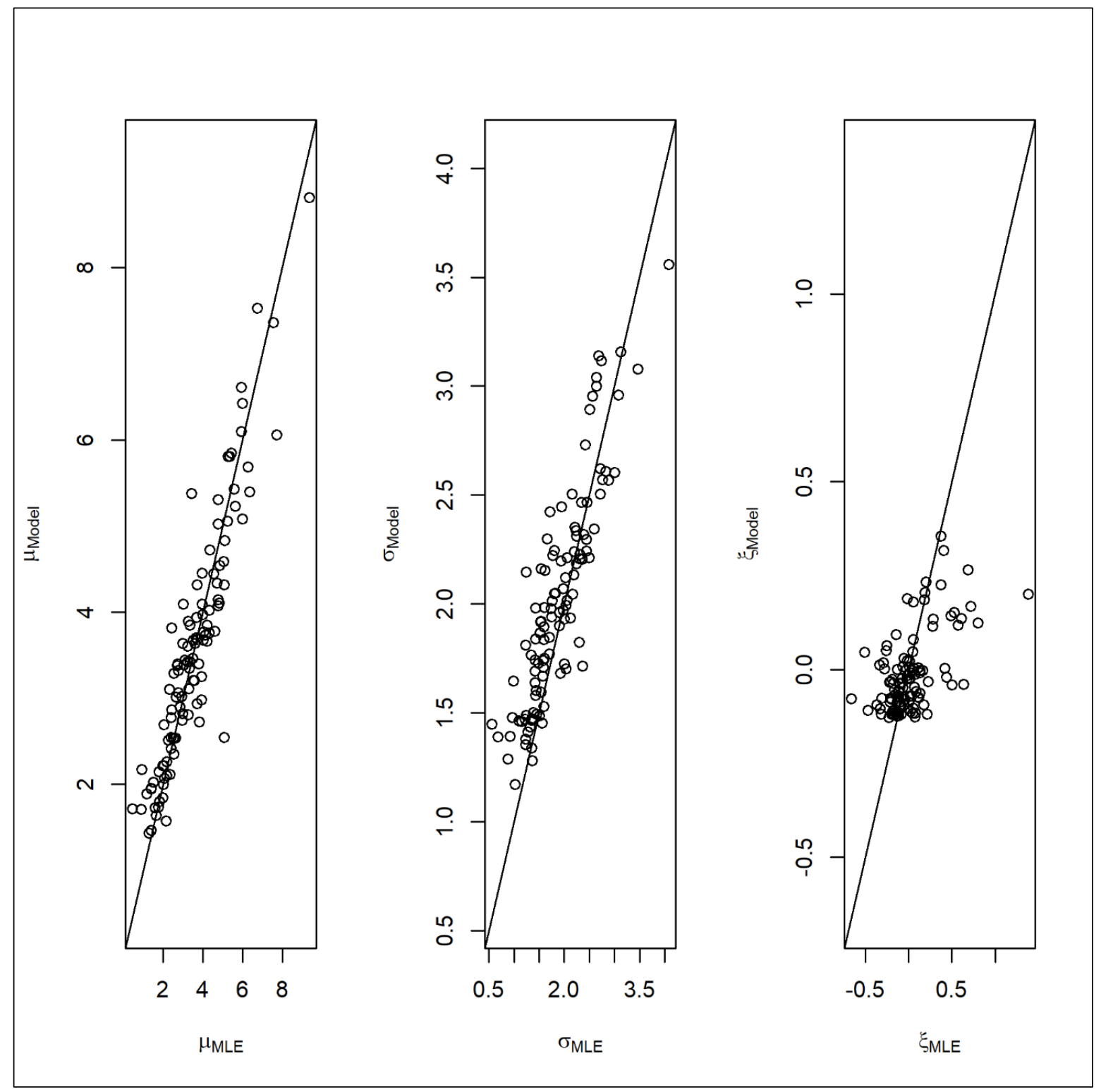


Figure 13. Fitted trend surfaces for (a) $\mu(x)$, (b) $\sigma(x)$, and (c) $\xi(x)$, and (d) the 100year pointwise return levels of SWE AM, in inches, for the upper CRB.

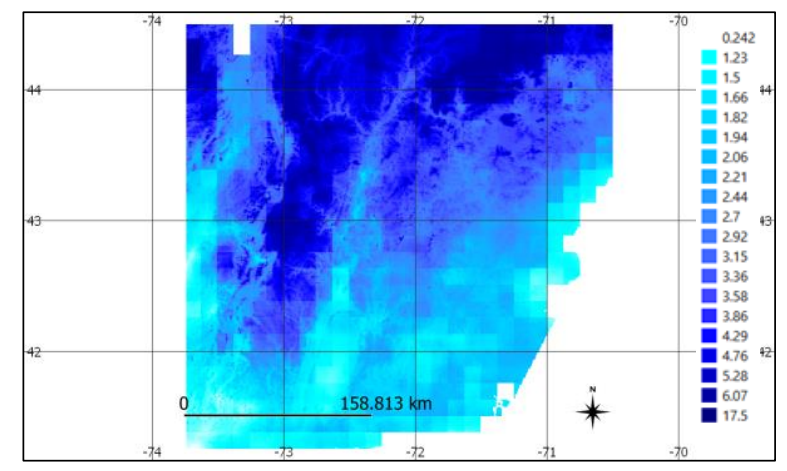

(a)

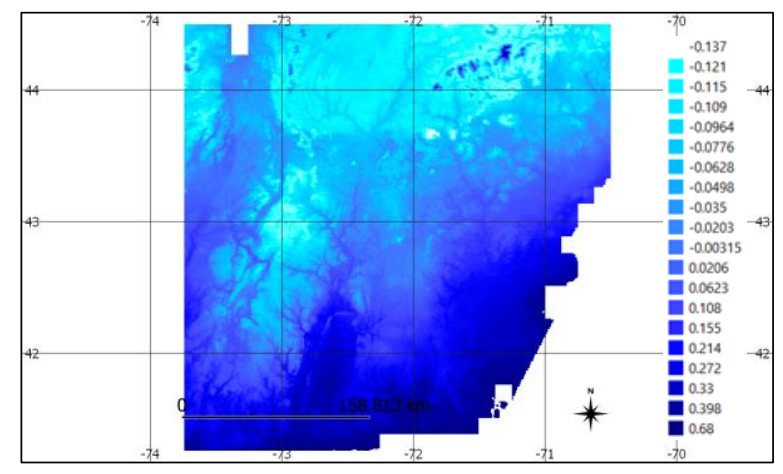

(c)

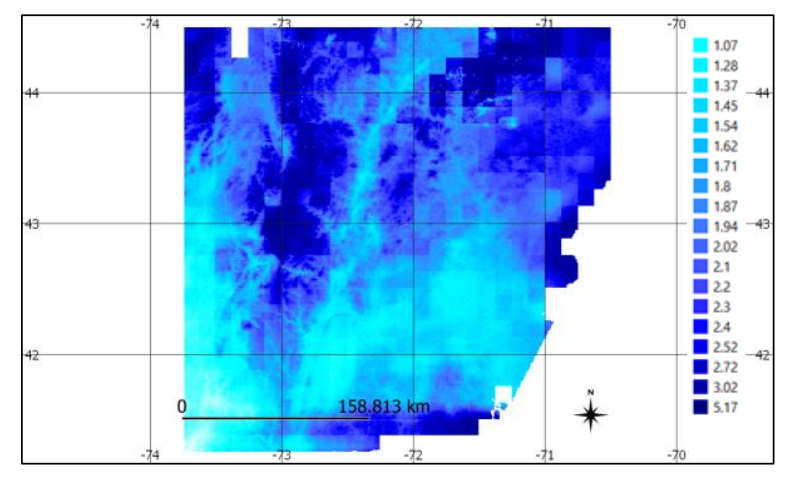

(b)

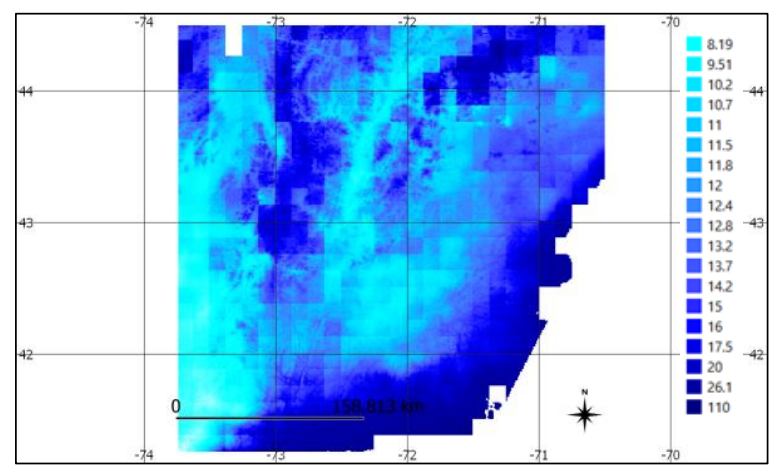

(d)

Figure 14. Two independent copies of the fitted max-stable model of SWE AM, in inches, for the White River Basin in the upper CRB.
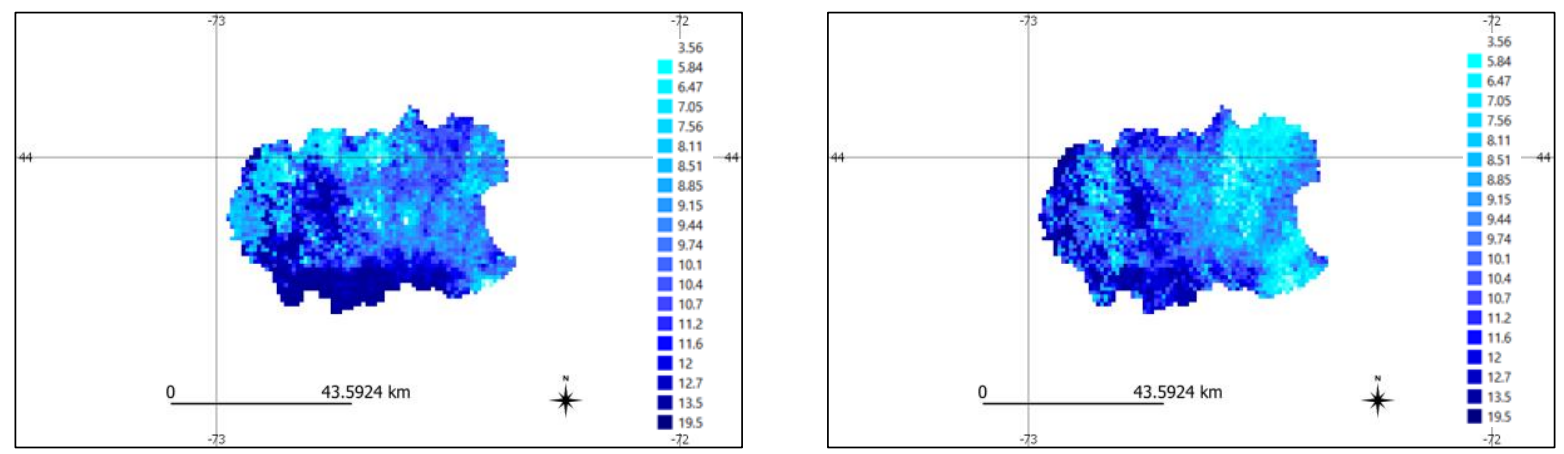
Figure 15. Computed histogram of mean basin SWE $A M$, in inches, for the White River Basin of the upper

CRB.

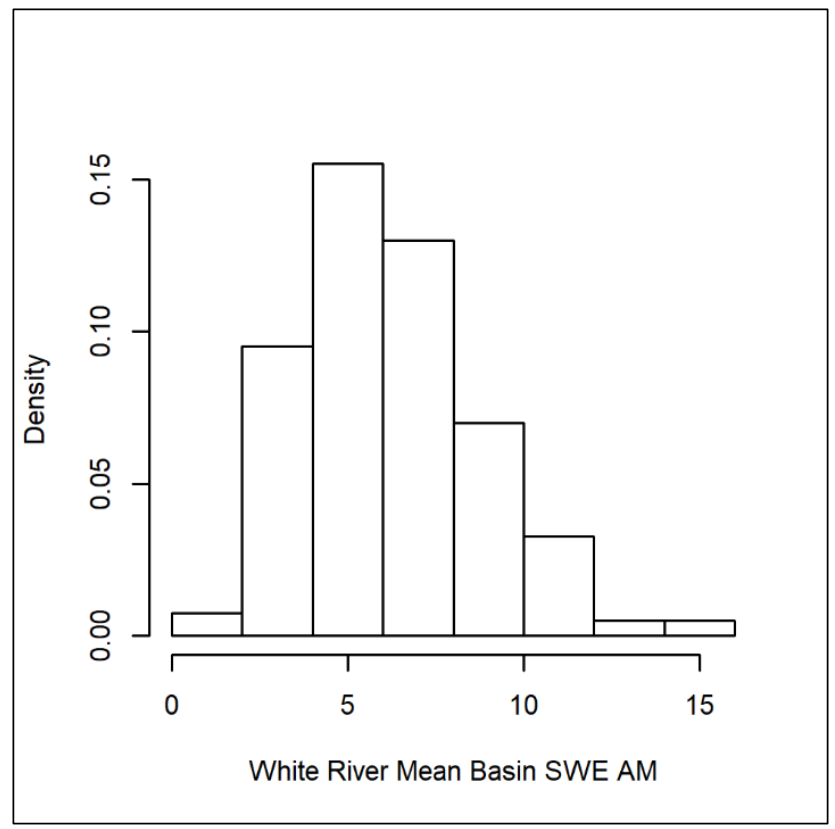

Table 6. Areal-based exceedance probability estimates of the SNODAS modeled mean SWE AM for the White River.

\begin{tabular}{|c|c|c|}
\hline Year & $\begin{array}{c}\text { White River } \overline{\boldsymbol{S W E}}_{\text {max }} \\
\text { (in) }\end{array}$ & $\begin{array}{c}\text { Exceedance } \\
\text { Probability }\end{array}$ \\
\hline 2004 & 5.33 & 0.585 \\
\hline 2005 & 6.79 & 0.325 \\
\hline 2006 & 3.14 & 0.945 \\
\hline 2007 & 7.12 & 0.290 \\
\hline 2008 & 11.09 & 0.030 \\
\hline 2009 & 7.97 & 0.225 \\
\hline 2010 & 5.89 & 0.500 \\
\hline 2011 & 9.17 & 0.120 \\
\hline 2012 & 4.52 & 0.725 \\
\hline 2013 & 5.25 & 0.610 \\
\hline 2014 & 8.63 & 0.180 \\
\hline 2015 & 7.30 & 0.265 \\
\hline 2016 & 2.64 & 0.970 \\
\hline 2017 & 5.88 & 0.505 \\
\hline
\end{tabular}




\section{Summary}

Practice-driven hydrologic modeling planning analyses involving the treatment of cool-season processes often require areal SWE estimates as input. Thus, pointwise SWE data observations must first be modeled, spatially, to properly support risk-informed hydrologic analyses involving the treatment of cool season processes. This case study application succinctly profiled a max-stable spatial process modeling analysis of SWE $\mathrm{AM}$ data within and surrounding the upper CRB as a means by which to address this identified need. The case study leveraged EVT and recent advances for regularizing linear models to systematically and efficiently guide the trend surface modeling component of the max-stable process deployment. Spatial dependence of the SWE AM data was observed to be non-trivial for the entire length of the modeled upper CRB, further underscoring the max-stable spatial process modeling analysis. Simulated independent copies of the fitted max-stable spatial process provided the basis to compute the distribution of mean SWE AM for the 690 square miles. White River Basin located in the upper CRB. The estimated distribution was subsequently used to evaluate the exceedance probability of SNODAS modeled mean SWE AM for the White River for the years 2004-2017, inclusive. The max-stable modeling analysis profiled in this case study is the spatial process extension of univariate and multivariate extreme value theory; hence, extrapolation from fitted max-stable processes are credible. Moreover, the max-stable modeling analysis profiled in this case study is general in that it can be applied to other hydrometeorological parameters of relevance for probabilistic flood hazard analyses. 


\section{References}

Blanchet, Juliette, and Anthony C. Davison. "Spatial Modeling of Extreme Snow Depth." Ann. Appl. Stat. 5(2011)(3): 1699-1725. doi:10.1214/11-AOAS464 https://projecteuclid.org/euclid.aoas/1318514282

Blanchet, J. 2009. Max-Stable Processes and Annual Maximum Snow Depth, 6th International Conference on Extreme Value Analysis, June 23-26, 2009, Fort Collins, CO, United States.

Brown, B. M., and S. I. Resnick. 1977. "Extreme Values of Independent Stochastic Processes.” Journal of Applied Probability 14:732-739.

Coles, Stuart. 2001. An Introduction to Statistical Modeling of Extreme Values. London: Springer-Verlag.

Cooley D., J. Cisewski, R. Erhardt, S. Jeon, E. Mannshardt, B. Omolo, and Y. Sun. 2012. "A Survey of Spatial Extremes: Measuring Spatial Dependence and Modeling Spatial Effects.” RevStat, 10:135-165.

Cooley, D., P. Naveau, and P. Poncet. 2006. "Variograms for Spatial Max-Stable Random Fields.” Lect. Notes Statist. 187: 373-390.

Davison, A., S. Padoan, and M. Ribatet. 2012. "Statistical Modelling Of Spatial Extremes." Statistical Science 27(2): 161-186.

Davison, A. C., and M. M. Gholamrezaee. 2011. Proc. R. Soc. A 2011. Published 12 October 2011. doi: 10.1098/rspa.2011.0412.

de Haan, L. 1984. “A Spectral Representation for Max-Stable Processes.” The Annals of Probability 12(4): 1194-1204.

Friedman, Jerome, Trevor Hastie, and Rob Tibshirani. 2010. "Regularization Paths for Generalized Linear Models via Coordinate Descent.” Journal of Statistical Software 33(1): 1-22.

Gareth James, Daniela Witten, Trevor Hastie, and Robert Tibshirani. 2013. An Introduction to Statistical Learning: With Applications in R. New York: Springer.

Hastie T., R. Tibshirani, and J. Friedman. 2009. The Elements of Statistical Learning: Prediction, Inference and Data Mining, 2nd edition. New York: Springer-Verlag.

Hastie, Trevor, and Junyang Qian. 2016. An Introduction to glmnet. https://glmnet.stanford.edu/articles/glmnet.html

Hoerl, A. E., and R. W. Kennard. 1970. "Ridge Regression: Biased Estimation for Nonorthogonal Problems.” Technometrics 12(1): 55-67.

Kabluchko, Z., M. Schlather, and L. de Haan. 2009. "Stationary Max-Stable Fields Associated to Negative Definite Functions.” Annals of Probability 37(5): 20422065 . 
Nicolet, Gilles, Nicolas Eckert, Samuel Morin, and Juliette Blanchet. 2015. Inferring Spatio-temporal Patterns in Extreme Snowfall in the French Alps Using Maxstable Processes, Procedia Environmental Sciences, Vol. 26. ISSN 1878-0296. https://doi.org/10.1016/j.proenv.2015.05.018

Olinda, R. A., J. Blanchet, C. A. C. dos Santos, V. A. Ozaki, and P. J. Ribeiro Jr. 2014. "Spatial Extremes Modeling Applied to Extreme Precipitation Data in the State of Paraná.” Hydrol. Earth Syst. Sci. Discuss. 11: 12731-12764. https://doi.org/10.5194/hessd-11-12731-2014.

Opitz, T. 2013. "Extremal-t Process: Elliptical Domain of Attraction and a Spectral Representation.” Journal of Multivariate Analysis 122:409-413.

Reich, Brian J., and Benjamin A. Shaby. 2012. "A Hierarchical Max-Stable Spatial Model for Extreme Precipitation.” Ann. Appl. Stat. 6(4): 1430-1451. doi:10.1214/12AOAS591. https://projecteuclid.org/euclid.aoas/1356629046

Ribatet M. 2009. A User's Guide to the SpatialExtremes Package. École Polytechnique Fédéral de Lausanne, Switzerland.

Ribatet, M. 2013. "Spatial Extremes: Max-Stable Processes at Work.” Journal of Société Française de Statistique (special edition on extreme value theory) 154(2): 156177.

Ribatet, M. 2015. Modelling Spatial Extremes with the Spatial Extremes Package. 9th International Conference on Extreme Value Analysis: EVA 2015. 15 June-19 June, Ann Arbor, MI.

Ribatet, M. 2017. “Modelling Spatial Extremes Using Max-Stable Processes.” Nonlinear and Stochastic Climate Dynamics. Edited by C. Franzke and T. O'Kane, 369-391. Cambridge University Press.

Ribatet, M. 2018. SpatialExtremes: Modelling Spatial Extremes. R Package Version 2.0-7. https://CRAN.R-project.org/package=SpatialExtremes

Ribatet, M., C. Dombry, and M. Oesting. 2015. "Spatial Extremes and Max-Stable Processes." Extreme Value Modeling and Risk Analysis: Methods and Applications. Edited by D. K. Dey and J. Yan. New York: Chapman and Hall.

Schlather, M. 2002. "Models for Stationary Max-Stable Random Fields.” Extremes 5(1):33-44.

Schlather, M., and J. Tawn. 2003. "A Dependence Measure for Multivariate and Spatial Extremes: Properties and Inference.” Biometrika 90(1):139-156.

Sexstone, G., and S. Fassnacht. 2014. "What Drives Basin Scale Spatial Variability of Snowpack Properties in Northern Colorado?” The Cryosphere 8(10.5194/tc-8): 329-2014.

Simon, Noah, Jerome Friedman, Trevor Hastie, and Rob Tibshirani. 2011. "Regularization Paths for Cox's Proportional Hazards Model via Coordinate Descent.” Journal of Statistical Software 39(5): 1-13. 
Smith, R. L. 1990. Max-Stable Processes and Spatial Extremes. http://scholar.google.com/scholar_url?url=https://www.researchgate.net/profile/Stilian_Stoev 2/publication/271095588_Upper_bounds_on_value-atrisk for the maximum portfolio_loss/links/564b824b08aeab8ed5e7694a.pdf\&hl=en\&sa=X\& scisig=AAGBfm0TFIhKIbfLArNGvU-AkgKamhRZGQ\&nossl=1\&oi=scholarr

Stephenson, Alec G., Eric Lehmann, and Aloke Phatak. 2016. "A Max-Stable Process Model for Rainfall Extremes at Different Accumulation Durations." Weather and Climate Extremes 13(September 2016): 44-53.

Takeuchi, K. 1976 "Distribution of Informational Statistics and a Criterion of Fitting [in Japanese]. Suri-Kagaku 153: 12-18.

Tibshirani, R. 1996. "Regression Shrinkage and Selection via the Lasso." J. Royal. Statist. Soc B. 58(1): 267-288).

Tibshirani, Robert, Jacob Bien, Jerome Friedman, Trevor Hastie, Noah Simon, Jonathan Taylor, and Ryan J. Tibshirani. 2010. "Strong Rules for Discarding Predictors in Lasso-type Problems.” Journal of the Royal Statistical Society: Series B (Statistical Methodology) 74(2): 245-266.

Tibshirani, R., J. Bien, J. Friedman, T. Hastie, N. Simon, J. Taylor, and R. Tibshirani. 2012. "Strong Rules for Discarding Predictors in Lasso-type Problems." JRSSB 74 .

Tikhonov, A. N. 1943. "On the Stability of Inverse Problems.” Doklady Akademii Nauk SSSR 39(5): 195-198.

Zou, H, and T. Hastie. 2005. "Regularization and Variable Selection via the Elastic Net." Journal of the Royal Statistical Society B 67(2): 301-320. 


\section{Acronyms and Abbreviations}

$\begin{array}{ll}\text { AM } & \text { Annual maxima } \\ \text { CRB } & \text { Connecticut River Basin } \\ \text { DEM } & \text { digital elevation model } \\ \text { EVT } & \text { extreme value theory } \\ \text { GEV } & \text { generalized extreme value } \\ \text { HBEF } & \text { Hubbard Brook Experimental Forest } \\ \text { MSE } & \text { mean squared error } \\ \text { NHDES } & \text { New Hampshire Department of Environmental Services } \\ \text { SCAN } & \text { Soil Climate Analysis Network } \\ \text { SNODAS } & \text { SNOw Data Assimilation System } \\ \text { SWE } & \text { snow water equivalent } \\ \text { USACE } & \text { US Army Corps of Engineers }\end{array}$




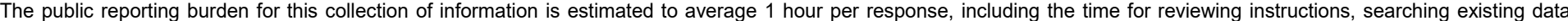

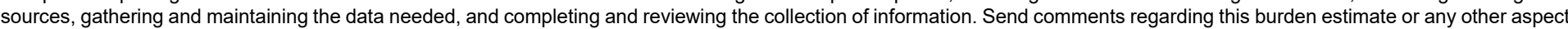

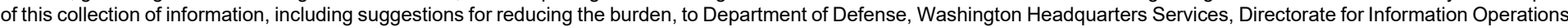

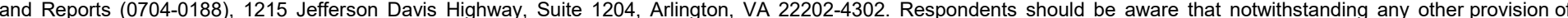
law, no person shall be subject to any penalty for failing to comply with a collection of information if it does not display a currently valid OMB control number. PLEASE DO NOT RETURN YOUR FORM TO THE ABOVE ADDRESS.

\begin{tabular}{l|l|l}
$\begin{array}{l}\text { 1. REPORT DATE } \\
\text { April } 2020\end{array}$ & $\begin{array}{l}\text { 2. REPORT TYPE } \\
\text { Final Report }\end{array}$ & 3. DATES COVERED (FrOm - To)
\end{tabular}

\section{TITLE AND SUBTITLE}

Analysis of Snow Water Equivalent Annual Maxima in the Upper Connecticut River Basin

Using a Max-Stable Spatial Process Model

5a. CONTRACT NUMBER

5b. GRANT NUMBER

5c. PROGRAM ELEMENT NUMBER

\section{AUTHOR(S)}

Brian Skahill, Joseph Kanney, and Meredith Carr 5d. PROJECT NUMBER

5e. TASK NUMBER

5f. WORK UNIT NUMBER

8. PERFORMING ORGANIZATION REPORT NUMBER Office of Nuclear Regulatory Research 11555 Rockville Pike Rockville, MD 20852-2738, Mail Stop

\section{ERDC/CHL TR-20-7} TWFN-10A12

\section{US Army Engineer Research and \\ Development Center \\ 3909 Halls Ferry Road \\ Vicksburg, MS 39180-6199}

7. PERFORMING ORGANIZATION NAME(S) AND

\section{SPONSORING/MONITORING AGENCY NAME(S) AND ADDRESS(ES)}

US Nuclear Regulatory Commission

11555 Rockville Pike

Rockville, MD 20852-2738

\section{DISTRIBUTION/AVAILABILITY STATEMENT}

Approved for public release; distribution is unlimited.

\section{SUPPLEMENTARY NOTES}

US NRC; MIPR Agreement No. NRC-HQ-60-14-I-0022

\section{ABSTRACT}

Recent advances from the science of spatial extremes and model regularization were applied to develop areal-based extremes of snow water equivalent (SWE) data for the upper Connecticut River Basin. Development of areal-based SWE exceedance probability estimates are of relevance for cool season probabilistic flood hazard analyses (PFHA). The approach profiled in this case study is applicable for other hydrometeor-ological variables of relevance to PFHA. The methodology conforms with Extreme Value Theory (EVT) for the analysis of spatial extremes; hence, there is a firm theoretical basis for extrapolation. Trend surface development is guided by EVT theory and recent advances for regularizing general linear models. $\mathrm{R}$, a free software environment for statistical computing and graphics, and QGIS, a free and open-source geographic information system, were the primary tools used for product development and delivery. The following R software packages were primarily used during project execution: evd, Glmnet, maps, raster, rgdal, SDMTools, sp, and SpatialExtremes. R software packages exist in the public domain and support PFHA analyses of varying complexities. Their application herein is not an endorsement or recommendation. It is recommended that one would need to evaluate any particular $\mathrm{R}$ software package regarding its suitability for use for any specific application.

\section{SUBJECT TERMS}

Connecticut River Watershed, Extreme value theory, Floods, Hydrologic models, Hydrology, Ice, Meltwater, Snow

\begin{tabular}{|c|c|c|c|c|c|}
\hline 16. SECURIT & CLASSIFICATIO & OF: & 17. LIMITATION OF & 18. NUMBER & 19a. NAME OF RESPONSIBLE PERSON \\
\hline a. REPORT & b. ABSTRACT & c. THIS PAGE & ABSTRACT & $\begin{array}{l}\text { OF } \\
\text { PAGES }\end{array}$ & Brian Skahill \\
\hline Unclassified & Unclassified & Unclassified & SAR & 42 & $\begin{array}{l}\text { 19b. TELEPHONE NUMBER (Include area code) } \\
\text { 971-804-0373 }\end{array}$ \\
\hline
\end{tabular}

